\title{
On the X-ray, optical emission line and black hole mass properties of local Seyfert galaxies ${ }^{\star}$
}

\author{
F. Panessa ${ }^{1}$, L. Bassani ${ }^{2}$, M. Cappi ${ }^{2}$, M. Dadina ${ }^{2}$, X. Barcons ${ }^{1}$, F. J. Carrera ${ }^{1}$, L. C. Ho ${ }^{3}$, and K. Iwasawa ${ }^{4}$ \\ 1 Instituto de Física de Cantabria (CSIC-UC), Avda. de los Castros, 39005 Santander, Spain \\ e-mail: panessa@ifca.unican.es \\ 2 INAF - IASF, via P. Gobetti 101, 40129 Bologna, Italy \\ The Observatories of the Carnegie Institution of Washington, 813 Santa Barbara St. Pasadena, CA 91101, USA \\ ${ }^{4}$ Max Planck Institut für Extraterrestrische Physik (MPE), Giessenbachstrasse 1, 85748 Garching, Germany
}

Received 23 January 2006 / Accepted 4 May 2006

ABSTRACT

\begin{abstract}
We investigate the relation between X-ray nuclear emission, optical emission line luminosities and black hole masses for a sample of 47 Seyfert galaxies. The sample, which has been selected from the Palomar optical spectroscopic survey of nearby galaxies (Ho et al. 1997a, ApJS, 112, 315), covers a wide range of nuclear powers, from $L_{2-10 \mathrm{keV}} \sim 10^{43} \mathrm{erg} / \mathrm{s}$ down to very low luminosities $\left(L_{2-10 \mathrm{keV}} \sim\right.$ $\left.10^{38} \mathrm{erg} / \mathrm{s}\right)$. Best available data from Chandra, XMM-Newton and, in a few cases, ASCA observations have been considered. Thanks to the good spatial resolution available from these observations and a proper modeling of the various spectral components, it has been possible to obtain accurate nuclear X-ray luminosities not contaminated by off-nuclear sources and/or diffuse emission. X-ray luminosities have then been corrected taking into account the likely candidate Compton thick sources, which are a high fraction ( $>30 \%$ ) among type 2 Seyferts in our sample. The main result of this study is that we confirm strong linear correlations between 2-10 $\mathrm{keV},[\mathrm{OIII}] \lambda 5007, \mathrm{H}_{\alpha}$ luminosities which show the same slope as quasars and luminous Seyfert galaxies, independent of the level of nuclear activity displayed. Moreover, despite the wide range of Eddington ratios $\left(L / L_{\mathrm{Edd}}\right)$ tested here (six orders of magnitude, from 0.1 down to $\sim 10^{-7}$ ), no correlation is found between the X-ray or optical emission line luminosities and the black hole mass. Our results suggest that Seyfert nuclei in our sample are consistent with being a scaled-down version of more luminous AGN.
\end{abstract}

Key words. accretion, accretion disks - X-rays: galaxies - galaxies: Seyfert - galaxies: nuclei

\section{Introduction}

The fundamental paradigm on which our understanding of Active Galactic Nuclei (AGN) activity is based is that the accretion of matter onto a super massive black hole (SMBH) powers the energy emission of these objects. Starting from this paradigm, the unification scheme (Unified Model, UM) proposed by Antonucci \& Miller (1985) relocates the various families of AGN within a single scenario in which the orientation of the emitter-absorber-observer system is responsible for the different spectral properties exhibited. In this scenario, type $2 \mathrm{ob}-$ jects are the ones in which the absorber (dusty torus) intercepts the line of sight, otherwise our view is directed into the nucleus and we are dealing with type 1 objects. Within the UM, the only other free parameter is, besides the orientation, the mechanism responsible for triggering strong radio emission and associated jets which are present in $\sim 10 \%$ of the AGN population.

In recent years, the number of cases in which the zerothorder UM predictions seem not to be completely adequate to explain all observational evidences is increasing, both in the local and in the distant universe. Type 1 AGN with significant absorption have been found (Cappi et al. 2006, hereafter C06, Mateos et al. 2005, Fiore et al. 2001a, etc.) as well as type 2 AGN without X-ray absorption (Caccianiga et al. 2004; Corral et al. 2005; Barcons et al. 2003; Panessa \& Bassani 2002; Pappa et al. 2001). In particular, it is not clear whether at very low luminosities the

\footnotetext{
* Appendix is only available in electronic form at
} http://www . edpsciences.org predictions of UM are still valid (Panessa \& Bassani 2002; Ho et al. 2001). The key for the comprehension of the whole AGN phenomenon seems to reside in a combination of the UM hypothesis and the fundamental parameters of AGN, such as black hole mass, Eddington ratio, and perhaps the black hole spin. After the discovery that SMBHs reside at the center of most, if not all, galaxies in the nearby universe (Kormendy \& Richstone 1995; Magorrian et al. 1998) and that a large fraction of them are active (at least 40\%, Ho et al. 1997b), it is of fundamental importance to understand the accretion physics in AGN and what triggers the different levels of activity.

One of the distinctive characteristic of nearby nuclei is their intrinsic faintness, i.e. $L_{\mathrm{Bol}}<10^{44} \mathrm{erg} / \mathrm{s}$ (Ho 2003), as well as their low level of activity; in terms of Eddington luminosity most of them have $L / L_{\mathrm{Edd}}<10^{-2}$ compared to $L / L_{\mathrm{Edd}} \sim 1$ of luminous AGN. Whether low luminosity AGN (LLAGN) are a scaled-down luminosity version of classical AGN or objects powered by different physical mechanism is a debated issue. It is not clear in fact, whether LLAGN are powered by radiatively inefficient accretion flows, such as advection dominated accretion flows (ADAF) and their variants (Narayan \& Yi 1994; Abramowicz 1997) instead of the standard geometrically thin optically thick accretion disk typically proposed as the accretion mechanism acting in the central regions of luminous AGN (Shakura \& Sunyaev 1973). LLAGN could also represent scaled up versions of black hole binaries in the steady-jet, hard X-ray state, as pointed out by the scaling relations reported in Merloni et al. (2003) and Falcke et al. (2004). On one hand, ADAF 
models are able to predict some of the spectral properties observed in many LLAGN, such as the lack of the "big blue bump" (Ho 1999). On the other hand, some LLAGN show properties which are common to luminous AGN, such as the observed correlations between optical emission lines and ionizing continuum (Ho \& Peng 2001) or X-ray emission (Ward et al. 1988; Terashima et al. 2000; Ho et al. 2001).

$\mathrm{X}$-rays are one of the most direct evidences of nuclear activity and are, therefore, fundamental to study the accretion processes. The new generation X-ray telescopes Chandra and XMM-Newton provide a much better spatial and spectral resolution than previous satellites. The high spatial resolution allows the detection of genuine low-luminosity AGN and the separation of the contribution of enhanced circumnuclear star formation from bona fide AGN with compact nuclear sources (Ho et al. 2001). The spectral resolution allows a good characterization of the X-ray spectral features. It is particularly important to determine the intrinsic absorbing column density accurately, since heavy obscuration suppresses the soft X-ray emission by a large factor and alters the spectral shape. As shown by the study of the distance-limited sample of nearby Seyfert galaxies (C06), the X-ray luminosity distribution is strongly affected by a nonnegligible number of Compton-thick sources $\left(N_{\mathrm{H}}>10^{24} \mathrm{~cm}^{-2}\right)$, which cannot be assessed by the data below $10 \mathrm{keV}$ alone.

Closely related to the theoretical and observational issues in LLAGN is the determination of the mass of the "massive dark objects", presumably black holes, located at the center of nearby galaxies, via stellar/gas kinematics (Gebhardt et al. 2003), via black hole mass $\left(M_{\mathrm{BH}}\right)$ and stellar velocity dispersion (Tremaine et al. 2002) or galaxy bulge mass (Richstone et al. 1998) correlations. The observed radiative output (e.g., X-ray luminosities) combined with $M_{\mathrm{BH}}$ estimates, allows us to measure the Eddington ratios and, therefore investigate the fundamental scaling of black hole properties with $M_{\mathrm{BH}}$ and accretion rate, $\dot{m}$.

We have chosen to investigate LLAGN and their relation with luminous AGN by studying the properties of a well defined sample of nearby Seyfert galaxies selected from Ho et al. (1997a), hereafter HFS97. In this paper, we focus on the X-ray, $\mathrm{H}_{\alpha}$ and [OIII] emission line properties. The estimates of the central BH masses are then used to test the activity levels of the sources. The strength of our approach resides in the capability to trace the absorption both via X-ray spectra and model independent diagnostics. For our analysis, we took advantage of the results presented in a companion paper (C06), where the XMMNewton data were presented for a distance limited sub-sample of the HFS97 Seyfert list.

The paper is organized as follows: a description of the sample is given in Sect. 2, details of the X-ray observations and data reduction can be found in Sect. 3, in Sect. 4 we discuss the fraction of heavily absorbed sources; relations between $\mathrm{X}$-ray/optical emission line luminosities with black hole masses are discussed in Sect. 6. The results and the effects of the incompleteness of the sample are discussed in Sect. 7. Finally, conclusions are summarized in Sect. 8. A description of the X-ray spectra analyzed in this work is deferred to the Appendix.

\section{The sample}

Our sample of Seyfert galaxies has been derived from the Palomar optical spectroscopic survey of nearby galaxies (Ho et al. 1995). From this survey, high-quality optical spectra of 486 bright $\left(B_{\mathrm{T}} \leq 12.5 \mathrm{mag}\right)$, northern $\left(\delta>0^{\circ}\right)$ galaxies have been taken and a comprehensive, homogeneous catalog of spectral classifications of all galaxies have been obtained (HFS97).
The Palomar survey is complete to $B_{\mathrm{T}}=12.0 \mathrm{mag}$ and $80 \%$ complete to $B_{\mathrm{T}}=12.5 \mathrm{mag}$ (Sandage et al. 1979). For the purpose of our study this is one of the best samples available up to now. In fact, it offers an accurate optical classification and the opportunity of detecting weak nuclei. Finally, the sample covers a large range of AGN luminosities $\left(L_{\mathrm{bol}} \sim 10^{41}-10^{44} \mathrm{erg} / \mathrm{s}\right)$ making it ideal for exploring possible trends with AGN power.

Here, the spectroscopic classification system of the Palomar survey is briefly summarized (see HFS97 for a more accurate description). The relative strength of the low-ionization optical forbidden lines ([O I] $\lambda \lambda 6300,6364,[\mathrm{~N} \mathrm{II}] \lambda \lambda 6548,6583$, [S II] $\lambda \lambda 6716,6731)$ compared to the hydrogen Balmer lines determines the classification of emission-line nuclei into two classes: H II nuclei (powered by stars) and AGN (powered by black-hole accretion). The separation between LINERs and Seyferts is instead given by the ratio $[\mathrm{OIII}]_{25007} / \mathrm{H}_{\beta}$ which corresponds to the ionization state of the narrow-line gas in AGN, i.e. $[\mathrm{OIII}]_{\lambda 5007} / \mathrm{H}_{\beta}<3$ for LINERs and $[\mathrm{OIII}]_{\lambda 5007} / \mathrm{H}_{\beta} \geq 3$ for Seyferts. Emission line nuclei having [OI] strengths intermediate between those of H II nuclei and LINERs are classified as "transition objects". Symbols used are: L = LINER, T = "transition object" (LINER + HII nucleus), and S = Seyfert. The classification in "type 1" or "type 2 " depends on the presence or absence of broad permitted lines. The measurement of the relative strength of the broad component of the hydrogen Balmer lines lead to subdivisions in the classification (type 1.0, 1.2, 1.5, 1.8 and 1.9; see Osterbrock 1981).

From the entire HFS97 sample we have extracted all Seyfert galaxies. The total sample of 60 Seyfert galaxies includes 39 type 2 (type 2 and 1.9 ) and 13 type 1 AGN (type 1.0, 1.2, 1.5). Eight objects, which are placed near the boundary between Seyfert and LINER, HII or transition classification, with a double classification (e.g., S2/T2, L2/S2, H/S2, etc.), have been included in the final sample. Hereafter we refer to these objects as "mixed Seyferts".

Seyfert galaxies classified as type 2 and 1.9 have been grouped into a more general "type 2" classification, while type 1.0, 1.2 and 1.5 have been grouped in the "type 1 " class. Type 2 and type 1.9 sources are normally both absorbed objects, while the type 1 group is referred to objects which are normally not affected by heavy absorption.

Two sources of the sample, NGC 4395 and NGC 4579, which have been classified by HFS97 as S1.8 and S1.9/L1.9 respectively, have been reclassified as type 1.5. A broad component is present in a number of optical (Filippenko \& Sargent 1989) and ultraviolet (Filippenko et al. 1993) emission lines of NGC 4395. Extremely broad permitted lines have been detected in NGC 4579. HST observations have revealed an $\mathrm{H}_{\alpha}$ component with FWZI of $\sim 18000 \mathrm{~km} \mathrm{~s}^{-1}$ (Barth et al. 1996 and Barth et al. 2001).

In objects like NGC 3608, NGC 3941, NGC 4472 and NGC 6482, the difficulty in the starlight subtraction process has lead to uncertainties in the classification (HFS97). Finally, the classification of NGC 185 is also uncertain, i.e., it is a dwarf spheroidal galaxy whose Seyfert-like line ratios maybe produced by stellar processes (Ho \& Ulvestad 2001).

Table 1 lists the properties of the host galaxies of the sample. Data for Cols. (2)-(8) are taken from the compilation of Ho \& Ulvestad (2001) and references therein. Distances for a few objects have been updated with more recent estimates (references are indicated in Col. (5)). The median distance of the sample galaxies is $25.7 \pm 17.7 \mathrm{Mpc}$. The nearest galaxy is NGC 185 (one of the companions of M 31) at $D=0.64 \mathrm{Mpc}$ and the farthest 
Table 1. The Seyfert galaxy sample.

\begin{tabular}{|c|c|c|c|c|c|c|c|}
\hline Name & $\begin{array}{c}\text { Seyfert } \\
\text { type } \\
(2)\end{array}$ & $\begin{array}{c}\text { Hubble } \\
\text { type } \\
(3)\end{array}$ & $\begin{array}{c}B \\
(\mathrm{mag}) \\
(4)\end{array}$ & $\begin{array}{c}\text { Distance } \\
(\mathrm{Mpc}) \\
(5)\end{array}$ & (6) & $\begin{array}{c}\text { RA } \\
(2000) \\
(7)\end{array}$ & $\begin{array}{c}\text { Dec } \\
(2000) \\
(8)\end{array}$ \\
\hline NGC 185 & $\mathrm{~S} 2$ & dE3pec & 10.10 & 0.64 & $\mathrm{a}$ & 003857.4 & +482014.4 \\
\hline NGC 676 & S2: & S0/a:spin & 10.50 & 19.5 & $\mathrm{~b}$ & 014857.4 & +055425.7 \\
\hline NGC 777 & $\mathrm{~S} 2 / \mathrm{L} 2$ & E1 & 12.49 & 66.5 & b & 020015.0 & +312545.5 \\
\hline NGC 1058 & S2 & $\mathrm{Sc}$ & 11.82 & 9.1 & $\mathrm{~b}$ & 024330.2 & +372027.2 \\
\hline NGC 1068 & S1.9 & $\mathrm{Sb}$ & 9.61 & 14.4 & $\mathrm{~b}$ & 024240.7 & -000047.6 \\
\hline NGC 1167 & $\mathrm{~S} 2$ & SO- & 13.38 & 65.3 & $b$ & 030142.4 & +351220.7 \\
\hline NGC 1275 & S1.5 & $\mathrm{Pec}$ & 12.64 & 70.1 & b & 031948.2 & +413042.4 \\
\hline NGC 1358 & $\mathrm{~S} 2$ & $\mathrm{SAB} 0 / \mathrm{a}$ & 13.04 & 53.6 & $\mathrm{~b}$ & 033339.7 & -0505 21.8 \\
\hline NGC 1667 & S2 & $\mathrm{SABc}$ & 12.77 & 61.2 & b & 044837.1 & -061911.9 \\
\hline NGC 2273 & S2 & $\mathrm{SBa}$ & 12.55 & 28.4 & $\mathrm{~b}$ & 065008.7 & +605045.0 \\
\hline NGC 2336 & $\mathrm{~L} 2 / \mathrm{S} 2$ & SABbc & 10.61 & 33.9 & b & 072703.8 & +801039.6 \\
\hline NGC 2639 & S1.9 & Sa? & 12.46 & 42.6 & b & 084338.0 & +501220.3 \\
\hline NGC 2655 & S2 & SAB0/a & 10.96 & 24.4 & b & 085538.8 & +781325.2 \\
\hline NGC 2683 & $\mathrm{~L} 2 / \mathrm{S} 2$ & $\mathrm{SAb}$ & 9.62 & 7.7 & $\mathrm{a}$ & 085241.7 & +332510.4 \\
\hline NGC 2685 & $\mathrm{~S} 2 / \mathrm{T} 2:$ & $\mathrm{SB} 0+$ pec & 12.12 & 16.2 & b & 085534.8 & +584401.6 \\
\hline NGC 3031 & S1.5 & $\mathrm{Sab}$ & 7.89 & 3.5 & $\mathrm{c}$ & 095533.2 & +690355.0 \\
\hline NGC 3079 & $\mathrm{~S} 2$ & SBc spin & 11.54 & 17.3 & d & 100158.5 & +554050.1 \\
\hline NGC 3147 & S2 & $\mathrm{Sbc}$ & 11.43 & 40.9 & b & 101653.3 & +732402.4 \\
\hline NGC 3185 & S2: & SB0/a & 12.99 & 21.3 & b & 101738.7 & +214117.2 \\
\hline NGC 3227 & S1.5 & SABa pec & 11.10 & 20.6 & b & 102330.6 & +195153.9 \\
\hline NGC 3254 & S2 & $\mathrm{Sbc}$ & 12.41 & 23.6 & b & 102919.9 & +292929.6 \\
\hline NGC 3486 & S2 & $\mathrm{SABc}$ & 11.05 & 7.4 & b & 110024.1 & +285831.6 \\
\hline NGC 3489 & $\mathrm{~T} 2 / \mathrm{S} 2$ & $\mathrm{SAB}+$ & 11.15 & 6.4 & $\mathrm{~b}$ & 110018.6 & +135404.0 \\
\hline NGC 3516 & $\mathrm{~S} 1.2$ & SB0 & 12.50 & 35.7 & b & 110647.5 & +723406.9 \\
\hline NGC 3608 & $\mathrm{~L} 2 / \mathrm{S} 2$ & E2 & 11.69 & 23.4 & $\mathrm{~b}$ & 111659.1 & +180854.6 \\
\hline NGC 3627 & $\mathrm{~T} 2 / \mathrm{S} 2$ & $\mathrm{SABb}$ & 9.13 & 6.6 & b & 112015.1 & +125921.6 \\
\hline NGC 3655 & $\mathrm{H} / \mathrm{S} 2$ & SAc & 12.08 & 26.5 & $\mathrm{~b}$ & 112254.7 & +163522.0 \\
\hline NGC 3735 & S2 & Sc: spin & 12.50 & 41.0 & b & 113557.5 & +703207.7 \\
\hline NGC 3941 & S2: & SB0 & 11.25 & 12.2 & a & 115255.4 & +365910.5 \\
\hline NGC 3976 & S2 & $\mathrm{SABb}$ & 12.30 & 37.7 & b & 115557.3 & +064457.0 \\
\hline NGC 3982 & S1.9 & SABb: & 11.78 & 20.5 & $\mathrm{e}$ & 115628.1 & +550730.6 \\
\hline NGC 4051 & S1.2 & SABbc & 10.83 & 17.0 & b & 120309.6 & +443152.8 \\
\hline NGC 4138 & S1.9 & $\mathrm{S} 0+$ & 12.16 & 13.8 & $\mathrm{a}$ & 120929.9 & +434106.0 \\
\hline NGC 4151 & S1.5 & SABab: & 11.50 & 20.3 & b & 121032.6 & +392420.6 \\
\hline NGC 4168 & S1.9: & E2 & 12.11 & 31.7 & $\mathrm{f}$ & 121217.3 & +131217.9 \\
\hline NGC 4169 & S2 & So & 13.15 & 50.4 & b & 121218.9 & +291044.0 \\
\hline NGC 4235 & $\mathrm{~S} 1.2$ & Sa spin & 12.62 & 35.1 & b & 121709.9 & +07 1129.1 \\
\hline NGC 4258 & S1.9 & SABbc & 9.10 & 7.2 & $\mathrm{a}$ & 121857.5 & +471814.3 \\
\hline NGC 4378 & $\mathrm{~S} 2$ & $\mathrm{Sa}$ & 12.63 & 35.1 & b & 122518.1 & +045531.6 \\
\hline NGC 4388 & S1.9 & Sb: spin & 11.76 & 16.7 & b & 122546.7 & +123940.9 \\
\hline NGC 4395 & $\mathrm{~S} 1 *$ & Sm: & 10.64 & 2.6 & b & 122548.9 & +333247.8 \\
\hline NGC 4472 & $\mathrm{~S} 2::$ & E2 & 9.37 & 16.7 & $\mathrm{~b}$ & 122946.8 & +075959.9 \\
\hline NGC 4477 & $\mathrm{~S} 2$ & SB0? & 11.38 & 16.8 & b & 123002.2 & +133811.3 \\
\hline NGC 4501 & S1.9 & $\mathrm{Sb}$ & 10.36 & 16.8 & $\mathrm{~b}$ & 123159.3 & +142513.4 \\
\hline NGC 4565 & S1.9 & $\mathrm{Sb}$ ? spin & 10.42 & 9.7 & $\mathrm{a}$ & 123621.1 & +255913.5 \\
\hline NGC 4579 & $\mathrm{~S} 1 *$ & $\mathrm{SABb}$ & 10.48 & 16.8 & $\mathrm{~b}$ & 123743.5 & +114904.9 \\
\hline NGC 4639 & S1.0 & SABbc & 12.24 & 22.9 & b & 124252.5 & +131524.1 \\
\hline NGC 4698 & S2 & Sab & 11.46 & 16.8 & b & 124822.9 & +082914.8 \\
\hline NGC 4725 & S2: & SABab pec & 10.11 & 13.2 & $\mathrm{a}$ & 125026.7 & +253002.3 \\
\hline NGC 5033 & S1.5 & $\mathrm{Sc}$ & 10.75 & 18.7 & b & 131327.5 & +363537.8 \\
\hline NGC 5194 & S2 & Sbc pec & 8.96 & 8.4 & b & 132952.4 & +471140.8 \\
\hline NGC 5273 & S1.5 & So & 12.44 & 16.5 & a & 134208.3 & +353915.2 \\
\hline NGC 5395 & $\mathrm{~S} 2 / \mathrm{L} 2$ & $\mathrm{Sb}$ pec & 12.10 & 46.7 & b & 135838.0 & +372528.3 \\
\hline NGC 5548 & S1.5 & $\mathrm{S} 0 / \mathrm{a}$ & 13.30 & 70.2 & b & 141759.5 & +2508 12.4 \\
\hline NGC 5631 & $\mathrm{~S} 2 / \mathrm{L} 2$ & So & 12.41 & 27.8 & $\mathrm{a}$ & 142633.3 & +563458.3 \\
\hline NGC 6482 & $\mathrm{~T} 2 / \mathrm{S} 2$ & $\mathrm{E}$ & 11.84 & 52.3 & $\mathrm{~b}$ & 175148.9 & +230419.1 \\
\hline NGC 6503 & $\mathrm{~T} 2 / \mathrm{S} 2$ & SAcd & 10.11 & 5.2 & $\mathrm{~g}$ & 174926.6 & +700840.1 \\
\hline NGC 6951 & S2 & SABbc & 11.64 & 24.1 & b & 203714.4 & +660619.7 \\
\hline NGC 7479 & S1.9 & $\mathrm{SBc}$ & 11.60 & 32.4 & $\mathrm{~b}$ & 230456.7 & +121923.2 \\
\hline NGC 7743 & S2 & SB0+ & 12.38 & 24.4 & $\mathrm{~b}$ & 234421.4 & +095603.6 \\
\hline
\end{tabular}

Notes: Col. (1): Galaxy name; Col. (2): optical classification, “*”= objects with a changed classification with respect to the original given by HFS97. The quality rating is given by ":" and "::" for uncertain and highly uncertain classification, respectively, as reported in HFS97; Col. (3): Hubble type; Col. (4) total apparent $B$ magnitude of the galaxy; Col. (5): galaxy distances; Col. (6): references on distances: (a) Tonry et al. (2001); (b) Tully (1988); (c) Paturel et al. (2002); (d) Cecil et al. (2002); (e) Stetson et al. (2001); (f) Merritt \& Ferrarese (2001); (g) Karachentsev \& Sharina (1997); Col. (7), (8) optical position in epoch J2000. 
is NGC 5548 at $D=70.2 \mathrm{Mpc}$, so we are sampling the local universe.

\section{The X-ray data}

An homogeneous and standard X-ray data analysis has been carried out on our selected Seyfert sample using Chandra and $X M M$-Newton observations. Chandra and XMM-Newton observations are available for 39 objects of the sample with 22 objects having observations with both observatories. Most of the XMMNewton observations are derived from an EPIC Guaranteed Time survey of a distance-limited sample of 27 Seyfert galaxies which have been published in a companion paper (C06) and we refer to that work for details.

To complement the X-ray information on the whole sample, a search in the literature for observations with previous X-ray observatories (operating in the $2-10 \mathrm{keV}$ energy range) has also been carried out. ASCA observations have been found for 8 further objects, references for those data taken from the literature are given in Table 2, except for NGC 3982 and NGC 4235 (briefly discussed in the Appendix) for which ASCA fluxes have been derived in this work. Adding all these data, 47 sources out of 60 objects have X-ray data available.

We used the CIAO 3.0 software for the Chandra data analysis to perform the data processing and calibration ${ }^{1}$. Starting from level 1 files, new level 2 event files were generated using the latest calibrations. We applied the pixel randomization introduced by the CXC (Chandra X-ray observatory Center) standard data processing (SDP) to avoid the instrumental "gridded" appearance of the data and any possible aliasing effects associated with this spatial grid. Finally, we examined light curves in order to clean the datasets for periods of anomalous background rates.

Most observations have been taken in the standard mode that allows a read-out mode of the full chip every $3.2 \mathrm{~s}$. For many bright sources in the sample more than one observation is often available. In this case, we have chosen the data set without gratings and, in order to minimize pile-up effects, the data set with $1 / 8$ or $1 / 2$ chip sub-array mode.

Other than the $\sim 250 \mathrm{ks}$ of EPIC Guaranteed Time for the distance-limited sample of 27 Seyfert galaxies, we further analyzed 5 public observations available from the XMM-Newton Science Archive (XSA) ${ }^{2}$. To be consistent with C06, we follow the same reduction and analysis procedure as described in that paper. Objects not belonging to the distance-limited survey of C06 are NGC 1275, NGC 3516, NGC 3227, NGC 5548 and NGC 7479 .

An atlas of Chandra and XMM-Newton images and spectra of the sources has been produced in the $0.3-10 \mathrm{keV}$ energy band (Panessa 2004). The results obtained indicate a high detection rate $(\sim 95 \%)$ of active nuclei, characterized, in $\sim 60 \%$ of the objects, also by the presence of nearby off-nuclear sources and/or, in $\sim 35 \%$ of the objects, diffuse emission. Altogether these results demonstrate that high spatial resolution is fundamental for this type of studies in order to isolate nuclear emission from other X-ray emitting components of the host galaxy.

Spectral analysis has been performed in order to first identify the underlying continuum when possible, then additional components and features have been included to best reproduce the data (following the same criteria as in C06). Each spectrum

\footnotetext{
1 All the data processing have been carried out following analysis threads on the Chandra web site:

http://cxc.harvard.edu/ciao/threads/index.html

${ }^{2}$ http://xmm.esac.esa.int/xsa
}

has been initially fitted with a model consisting of a power-law plus absorption fixed at the Galactic value and intrinsic absorption left free to vary. Some high quality spectra required more complex modeling and additional features (a soft component and/or emission line features). Details on the spectral analysis performed in this work are given source by source in the Appendix. X-ray fluxes have been obtained from the best spectral fit found and corrected for Galactic and intrinsic absorption (Col. 4, Table 2), except for those cases having very poor statistics for which a standard power-law model with a photon index fixed at the value of 1.8 and Galactic absorption was assumed. Luminosities were computed using $H_{0}=75 \mathrm{~km} \mathrm{~s}^{-1} \mathrm{Mpc}^{-1}$ and distances as in Table 2.

The distributions of spectral parameters, in particular for type 1 objects, are found to be within the range of values observed in luminous AGN. The observed distribution of the instrinsic column densities for the total sample ranges from the typical Galactic values $\left(\sim 10^{20} \mathrm{~cm}^{-2}\right)$ to very high absorptions (i.e. $\sim 10^{23} \mathrm{~cm}^{-2}$ ). Nearly $30 \%$ of type 1 Seyfert galaxies are characterized by a significant amount of absorption (i.e. $\geq 10^{22} \mathrm{~cm}^{-2}$ ) that could be ascribed to ionized material and/or dense gas clouds crossing the line of sight. The distribution of the column densities found for our type 2 Seyfert galaxies deviates from past results showing mostly mildly absorbed objects. However, it is well known that Compton thick sources (with $N_{\mathrm{H}}>10^{24} \mathrm{~cm}^{-2}$ ) may appear as objects with little absorption (Risaliti et al. 1999) if fitted with simple models and/or if only data with poor statistics are available. We compared between the column density distributions of type 1.9 and pure type $2 \mathrm{ob}-$ jects to find no difference (KS probability of 0.42 ). We therefore grouped the two classes into a single group.

Summarizing, the X-ray properties of our sample resemble those of more luminous AGN, except for four sources which have been only marginally detected in X-rays (NGC 1058, NGC 3627, NGC 3489 and NGC 4472). In this work we focus on the nuclear X-ray luminosities (Col. 4, Table 2) which are obtained in a homogeneous way and are as less contaminated as possible by the presence of diffuse emission and/or off-nuclear sources. The correction applied to the luminosities is discussed in the next section.

\section{How many heavily absorbed sources?}

X-ray spectra have proved to be an important tool to have a direct estimate of the amount of absorbing material in Seyfert galaxies. However, for values of the column density $>10^{24} \mathrm{~cm}^{-2}$, X-rays above a few $\mathrm{keV}$ are not able to penetrate the absorbing material and the photoelectric cutoff (if any) in the observed spectrum below $10 \mathrm{keV}$ does not provide information on the real column density and so the galaxy may be erroneously classified as a low-absorption object. This leads to an underestimation of the intrinsic hard X-ray luminosity heavily affecting the shape of the X-ray luminosity distribution of type 2 Seyferts (C06).

In order to have luminosities which are as much as possible not affected by absorption, we have searched within our sample for absorbed objects not classified as such from the X-ray modelling. To do so, we have taken advantage of some powerful diagnostic tools like the flux diagnostic diagrams (Bassani et al. 1999; Panessa \& Bassani 2002).

Flux diagnostic diagrams are based on measuring the X-ray luminosity against an isotropic indicator of the intrinsic brightness of the source to evaluate the true amount of absorption. If the UM is correct, a molecular torus should be present in Seyfert galaxies that could block the X-ray emission coming 
Table 2. Multi-wavelength luminosities and $M_{\mathrm{BH}}$ of the total X-ray sample.

\begin{tabular}{|c|c|c|c|c|c|c|c|c|c|c|c|}
\hline $\begin{array}{l}\text { Name } \\
\text { (1) }\end{array}$ & $\begin{array}{l}\text { Class } \\
\text { (2) }\end{array}$ & $\begin{array}{l}\text { Sat } \\
\text { (3) }\end{array}$ & $\begin{array}{c}\log F_{2-10 \mathrm{keV}} \\
\text { (4) }\end{array}$ & $\begin{array}{c}\log L_{2-10 \mathrm{keV}} \\
(5)\end{array}$ & $\begin{array}{l}\text { Ref. } \\
(6)\end{array}$ & $\begin{array}{l}\text { CThick } \\
\text { (7) }\end{array}$ & $\begin{array}{c}\log L_{[\text {[OIII] }} \\
(8)\end{array}$ & $\begin{array}{l}\log L_{\mathrm{H} \alpha} \\
\text { (9) }\end{array}$ & $\begin{array}{c}\log M_{\mathrm{BH}} \\
(10)\end{array}$ & $\begin{array}{l}\text { Method } \\
\text { (11) }\end{array}$ & $\begin{array}{l}\text { Ref. } \\
\text { (12) }\end{array}$ \\
\hline NGC 676 & S2: & $\mathrm{X}$ & -13.62 & 40.79 & 1 & $\sqrt{ }$ & 39.04 & 38.62 & & & \\
\hline NGC 1058 & S2 & $\mathrm{C}$ & $<-14.42$ & $<37.55$ & 2 & ? & 37.90 & 38.16 & 4.88 & $\mathrm{I}_{\sigma}$ & 8 \\
\hline NGC 1068 & S1.9 & $X$ & -11.31 & 42.84 & 1 & $\sqrt{ }$ & 41.91 & 41.53 & 7.20 & M & 1 \\
\hline NGC 1167 & $\mathrm{~S} 2$ & A & -13.39 & 42.07 & 3 & $\sqrt{ }$ & 40.76 & 40.81 & - & - & - \\
\hline NGC 1275 & S1.5 & X & -10.91 & 42.83 & 2 & $x$ & 41.91 & 42.07 & 8.51 & $\mathrm{I}_{\sigma}$ & 2 \\
\hline NGC 1667 & $\mathrm{~S} 2$ & A & -13.1 & 42.31 & 3 & $\sqrt{ }$ & 40.42 & 40.17 & 7.88 & $\mathrm{I}_{\sigma}$ & 2 \\
\hline NGC 2273 & S2 & A & -12.16 & 42.58 & 4 & $\sqrt{ }$ & 40.94 & 40.92 & 7.30 & $\mathrm{I}_{\sigma}$ & 2 \\
\hline NGC 2639 & S1.9 & A & -12.49 & 40.82 & 5 & $?$ & 40.40 & 40.56 & 8.02 & $\mathrm{I}_{\sigma}$ & 9 \\
\hline NGC 2655 & $\mathrm{~S} 2$ & A & -10.98 & 41.85 & 5 & $x$ & 39.92 & 40.03 & 7.77 & $\mathrm{I}_{\sigma}$ & 9 \\
\hline NGC 2683 & $\mathrm{~L} 2 / \mathrm{S} 2$ & $\mathrm{C}$ & -13.62 & 38.21 & 2 & ? & 38.58 & 37.69 & 7.51 & $\mathrm{I}_{\sigma}$ & 9 \\
\hline NGC 2685 & S2/T2: & X & $:-12.53$ & 39.94 & 1 & $x$ & 38.96 & 39.21 & 7.15 & $\mathrm{I}_{\sigma}$ & 9 \\
\hline NGC 3031 & S1.5 & X & -10.89 & 40.25 & 1 & $\times$ & 38.56 & 38.94 & 7.80 & $\mathrm{~S}$ & 1 \\
\hline NGC 3079 & $\mathrm{~S} 2$ & $\mathrm{C}$ & -11.69 & 42.62 & 2 & $\sqrt{ }$ & 40.07 & 40.89 & 7.65 & $\mathrm{I}_{\sigma}$ & 3 \\
\hline NGC 3147 & S2 & $\mathrm{C}$ & -11.39 & 41.89 & 2 & $x$ & 40.07 & 40.01 & 8.79 & $\mathrm{I}_{\sigma}$ & 3 \\
\hline NGC 3185 & S2: & $X$ & -13.7 & 40.79 & 1 & $\sqrt{ }$ & 39.90 & 40.07 & 6.06 & $\mathrm{I}_{\sigma}$ & 2 \\
\hline NGC 3227 & S1.5 & X & -10.94 & 41.74 & 1 & $x$ & 40.51 & 40.20 & 7.59 & $\mathrm{R}$ & 4 \\
\hline NGC 3486 & S2 & X & -12.93 & 38.86 & 1 & ? & 37.99 & 37.85 & 6.14 & $\mathrm{I}_{\sigma}$ & 8 \\
\hline NGC 3489 & $\mathrm{~T} 2 / \mathrm{S} 2$ & $\mathrm{C}$ & $<-13.57$ & $<39.88$ & 2 & $\sqrt{ }$ & 38.86 & 38.48 & 7.48 & $\mathrm{I}_{\sigma}$ & 5 \\
\hline NGC 3516 & $\mathrm{~S} 1.2$ & $\mathrm{C}$ & -10.87 & 42.29 & 2 & $x$ & 40.50 & 39.91 & 7.36 & $\mathrm{R}$ & 1 \\
\hline NGC 3608 & $\mathrm{~L} 2 / \mathrm{S} 2$ & $\mathrm{C}$ & -13.69 & 39.10 & 2 & $x$ & 38.22 & 38.23 & 8.04 & $\mathrm{~S}$ & 1 \\
\hline NGC 3627 & $\mathrm{~T} 2 / \mathrm{S} 2$ & $\mathrm{C}$ & $<-13.81$ & $<37.88$ & 2 & $?$ & 38.79 & 39.35 & 7.99 & $\mathrm{I}_{\sigma}$ & 5 \\
\hline NGC 3941 & S2: & $X$ & -13.35 & 38.88 & 1 & $x$ & 38.63 & 38.78 & 8.15 & $\mathrm{I}_{\sigma}$ & 7 \\
\hline NGC 3982 & S1.9 & A & -13.28 & 41.18 & 2 & $\sqrt{ }$ & 40.50 & 39.87 & 6.09 & $\mathrm{I}_{\sigma}$ & 2 \\
\hline NGC 4051 & S1.2 & $\mathrm{C}$ & -11.2 & 41.31 & 2 & $x$ & 39.81 & 39.68 & 6.11 & $\mathrm{R}$ & 4 \\
\hline NGC 4138 & S1.9 & $X$ & -11.04 & 41.29 & 1 & $x$ & 38.74 & 38.69 & 7.75 & $\mathrm{I}_{\sigma}$ & 9 \\
\hline NGC 4151 & S1.5 & $X$ & -10.2 & 42.47 & 1 & $x$ & 41.47 & 40.94 & 7.18 & $\mathrm{R}$ & 4 \\
\hline NGC 4168 & S1.9: & X & -13.19 & 39.87 & 1 & $x$ & 38.46 & 38.93 & 7.95 & $\mathrm{I}_{\sigma}$ & 6 \\
\hline NGC 4235 & S1.2 & A & -10.92 & 42.22 & 2 & $x$ & 40.41 & 40.97 & - & - & - \\
\hline NGC 4258 & S1.9 & $\mathrm{C}$ & -10.91 & 40.86 & 2 & $x$ & 39.07 & 38.65 & 7.61 & M & 1 \\
\hline NGC 4388 & S1.9 & $\mathrm{C}$ & -10.78 & 41.72 & 2 & $x$ & 40.54 & 40.26 & 6.80 & $\mathrm{I}_{\sigma}$ & 3 \\
\hline NGC 4395 & S1 & $X$ & -11.07 & 39.81 & 1 & $x$ & 38.28 & 38.53 & 5.04 & $\mathrm{~S}$ & 1 \\
\hline NGC 4472 & $\mathrm{~S} 2::$ & $\mathrm{C}$ & $<-13.18$ & $<39.32$ & 2 & $x$ & $<37.81$ & $<37.59$ & 8.80 & $\mathrm{I}_{\sigma}$ & 6 \\
\hline NGC 4477 & $\mathrm{~S} 2$ & $X$ & -12.85 & 39.65 & 1 & $x$ & 39.04 & 39.06 & 7.92 & $\mathrm{I}_{\sigma}$ & 9 \\
\hline NGC 4501 & S1.9 & $X$ & -12.91 & 39.59 & 1 & $x$ & 39.23 & 39.06 & 7.90 & $\mathrm{I}_{\sigma}$ & 3 \\
\hline NGC 4565 & S1.9 & $X$ & -12.6 & 39.43 & 1 & $x$ & 38.69 & 38.72 & 7.70 & $\mathrm{I}_{\sigma}$ & 3 \\
\hline NGC 4579 & $\mathrm{~S} 1$ & $\mathrm{C}$ & -11.47 & 41.03 & 6 & $x$ & 39.42 & 39.72 & 7.78 & $\mathrm{I}_{\sigma}$ & 3 \\
\hline NGC 4639 & S1.0 & $X$ & -12.55 & 40.22 & 1 & $x$ & 38.71 & 39.90 & 6.85 & $\mathrm{I}_{\sigma}$ & 8 \\
\hline NGC 4698 & $\mathrm{~S} 2$ & $X$ & -13.34 & 39.16 & 1 & $x$ & 38.86 & 38.74 & 7.84 & $\mathrm{I}_{\sigma}$ & 9 \\
\hline NGC 4725 & S2: & $\mathrm{X}$ & -13.4 & 38.89 & 1 & $x$ & 38.58 & 38.24 & 7.49 & $\mathrm{I}_{\sigma}$ & 3 \\
\hline NGC 5033 & S1.5 & $X$ & -11.52 & 41.08 & 1 & $x$ & 39.72 & 40.42 & 7.30 & $\mathrm{I}_{\sigma}$ & 3 \\
\hline NGC 5194 & $\mathrm{~S} 2$ & $\mathrm{C}$ & -12.77 & 40.91 & 2 & $\sqrt{ }$ & 39.90 & 39.87 & 6.95 & $\mathrm{I}_{\sigma}$ & 2 \\
\hline NGC 5273 & S1.5 & $\mathrm{X}$ & -11.13 & 41.36 & 1 & $x$ & 39.03 & 38.48 & 6.51 & $\mathrm{I}_{\sigma}$ & 2 \\
\hline NGC 5548 & S 1.5 & $X$ & -10.5 & 43.25 & 2 & $x$ & 41.16 & 40.26 & 8.03 & $\mathrm{I}_{\sigma}$ & 4 \\
\hline NGC 6482 & $\mathrm{~T} 2 / \mathrm{S} 2$ & $\mathrm{C}$ & -13.33 & 40.16 & 2 & $x$ & - & 39.26 & 8.75 & $\mathrm{I}_{\sigma}$ & 9 \\
\hline NGC 6503 & $\mathrm{~T} 2 / \mathrm{S} 2$ & $\mathrm{C}$ & -14.39 & 37.10 & 2 & ? & - & 37.41 & 5.56 & $\mathrm{I}_{\sigma}$ & 8 \\
\hline NGC 7479 & S1.9 & $\mathrm{X}$ & -11.95 & 41.12 & 2 & ? & 40.25 & 40.67 & 7.07 & $\mathrm{I}_{\sigma}$ & 9 \\
\hline NGC 7743 & $\mathrm{~S} 2$ & A & -13.14 & 41.47 & 5 & $\sqrt{ }$ & 40.21 & 40.24 & 6.59 & $\mathrm{I}_{\sigma}$ & 2 \\
\hline
\end{tabular}

Notes: Col. (1): galaxy name; Col. (2): optical classification; Col. (3): Satellite used: "C"=Chandra, "X"=XMM-Newton, "A"=ASCA; Col. (4): logarithm of the 2-10 keV flux in units of erg cm $\mathrm{cm}^{-2} \mathrm{~s}^{-1}$, not corrected for Compton thick candidates. Col. (5): logarithm of the 2-10 keV luminosity, corrected for Compton thick candidates. Col. (6): X-RAY REFERENCES: (1) Cappi et al. (2006); (2) this work; (3) Pappa et al. (2001); (4) Guainazzi et al. (2005); (5) Terashima et al. (2002); (6) Eracleous et al. (2002); Col. (7): Final classification: “ $\sqrt{\prime \prime}=$ Compton thick candidates, " $\times$ "=Compton thin candidate and "?"=doubtful objects.; Col. (8): logarithm of the $[\mathrm{OIII}]_{\text {} 5007}$ luminosity corrected for Galactic absorption and NLR extinction; Col. (9): logarithm of the $\mathrm{H} \alpha$ (broad+narrow component) luminosity corrected for Galactic absorption and NLR extinction; Col. (10): logarithm of the $M_{\mathrm{BH}}$ value in units of $M_{\odot}$; Col. (11): $M_{\mathrm{BH}}$ measurement methods; M: maser kinematics; G: gas kinematics; S: stellar kinematics; R: reverberation mapping; $\mathrm{I}_{\sigma}$ : inferred from the mass-velocity dispersion correlation; Col. (12): $M_{\mathrm{BH}}$ references: (1) Ho (2002); (2) Woo \& Urry (2002); (3) Merloni et al. (2003); (4) Kaspi et al. (2000); (5) Pellegrini (2005); (6) Merritt \& Ferrarese (2001); (7) di Nella et al. (1995); (8) Barth et al. (2002); (9) McElroy (1995).

from the central engine when it intercepts the line of sight. However, emission coming from larger scales, like the Narrow Line Region or a non nuclear starburst region, should not be affected by obscuration. The $[\mathrm{OIII}]_{\lambda 5007}$ emission line is thought to originate in narrow line regions by UV ionizing photons produced by the AGN. It has been shown in the literature that the distribution of [OIII $]_{\lambda 5007}$ flux is similar in both types of Seyfert (Mulchaey et al. 1994); also the NLR size determined from HST data is similar in type 1 and type 2 Seyferts (Schmitt et al. 2003). Although the physics occurring in the NLR is very complex and the observed luminosity depends on physical and geometrical properties, for example on the opening angle, the $[\mathrm{OIII}]_{\lambda 5007}$ flux has been extensively used as an isotropic indicator of the intrinsic AGN power (Maiolino \& Rieke 1995; Risaliti et al. 1999; Bassani et al. 1999, Guainazzi et al. 2005, C06, Heckman 2005). The relation between the $[\mathrm{OIII}]_{\lambda 5007}$ and the X-ray luminosities 


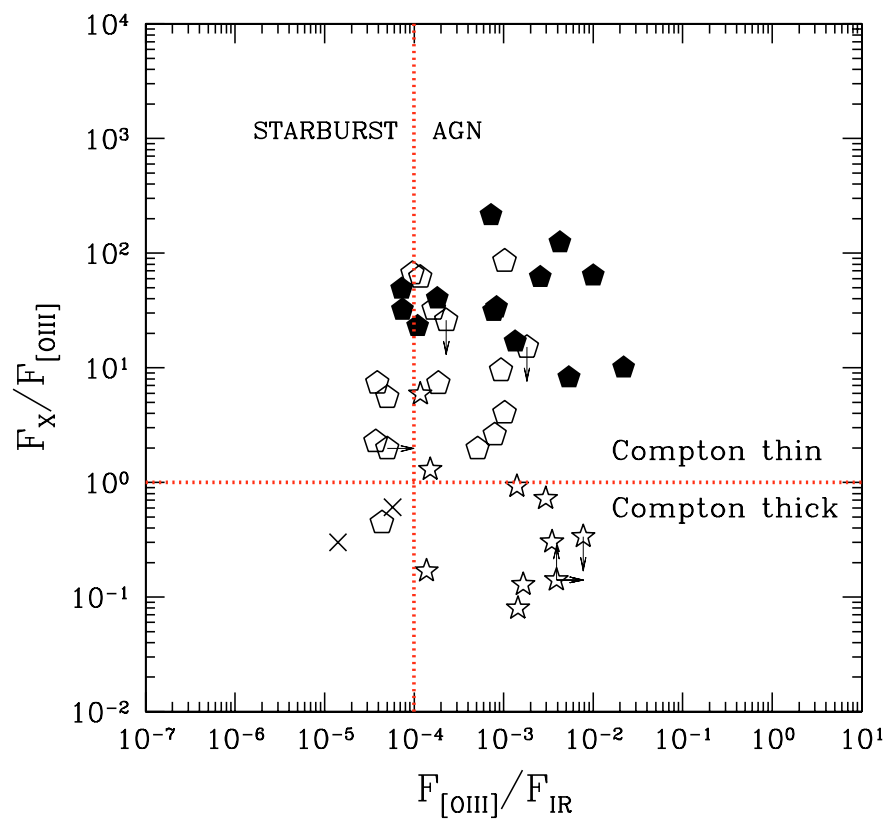

Fig. 1. $F_{\mathrm{X}} / F_{[\mathrm{OIII}]}$ vs. $F_{[\mathrm{OIII}]} / F_{\mathrm{IR}}$ diagram. Compton thin, Compton thick and starburst regions have been separated by dashed lines. Type 1 objects are plotted as filled polygons, type 2 as empty polygons, "mixed Seyfert" objects as crosses and Compton thick candidates as stars.

has also been recently studied for a sample of broad- and narrowline Seyfert 1 galaxies (Kraemer et al. 2004); these authors found that the X-ray/[OIII] ratio can be lowered as a result of the $\mathrm{X}$ ray absorption. Another frequently used isotropic indicator is the far-infrared emission (Mulchaey et al. 1994; Mas-Hesse et al. 1994), probably produced in the coolest regions of the molecular torus or even over a larger scale; note however that infrared emission could be anisotropic at short wavelengths $<12 \mu \mathrm{m}$ in heavily absorbed objects.

The $F_{\mathrm{X}} / F_{[\mathrm{OIII}]}$ and $F_{[\mathrm{OIII}]} / F_{\mathrm{IR}}$ ratio can provide an independent way to establish which is the dominant component between AGN or starburst and at the same time it is a powerful tool in the detection of Compton thick sources when an X-ray spectral analysis is not sufficient (Panessa \& Bassani 2002).

A flux diagram is presented in Fig. 1. All far-infrared fluxes are based on IRAS data derived from HFS97. The [OIII] $\lambda 5007$ flux of each galaxy has been corrected for extinction (HFS97). Type 1 objects are plotted as filled polygons, type 2 as empty polygons and "mixed Seyferts" as crosses. Compton thick candidates, as discussed below, have been marked with stars. Discriminating values, chosen for SB (starburst), Compton thick and Compton thin regions, are given as in Panessa \& Bassani (2002).

Most of the sources of both types lie in the AGN region and only a small fraction are located at the boundary between AGN and Starburst. Indeed, the X-ray analysis of most of our sample objects has shown that they host an AGN. The positions of the few borderline objects in our diagram could be due to the low IRAS angular resolution ${ }^{3}$, i.e. the presence of enhanced star-forming regions in the host galaxy may contribute significantly to increase the IR flux in these Seyferts; this translates into $F_{[\mathrm{OIII}]} / F_{\mathrm{IR}}$ ratios smaller than that of the AGN alone. For example, NGC 5033 is known to host bright HII regions in the

\footnotetext{
${ }^{3}$ Note that infrared data have been taken using a beam size of $\sim 8^{\prime}$, while optical and X-ray data are derived using much smaller apertures, i.e. $\sim 1^{\prime \prime}$ and $\sim 2^{\prime \prime}-25^{\prime \prime}$ respectively.
}

inner parts of its spiral arms (Pérez García \& Rodríguez Espinosa 1998); in NGC 4639 (González-Delgado et al. 1997) as well as in NGC 4725 (Sandage \& Bedke 1994) many bright HII regions have been observed, while NGC 3031 is known to host a very strong underlying stellar continuum (Alonso-Herrero et al. 2000).

In order to identify Compton thick sources, the $F_{\mathrm{X}} / F_{\text {[OIII] }}$ ratio has been considered: the most populated region is that of Compton thin AGN where all broad line Seyferts and 17 type 2 objects are located while the remaining type 2 Seyferts lay in the Compton thick region. As a matter of fact, four out of six known Compton thick sources, namely NGC 1068, NGC 1667, NGC 2273 and NGC 5194, occupy this region confirming the reliability of this diagnostic diagram, while NGC 3079, a Compton thick source, is above the Compton thick boundary, probably because of the presence of filaments in its nuclear region whose emission contributes significantly in the $0.1-6.5 \mathrm{keV}$ range (Cecil et al. 2002). There is a group of Compton-thick source candidates, namely NGC 676, NGC 1167, NGC 3185, NGC 3982 and NGC 7743, for which $\mathrm{X}$-ray spectral analysis alone cannot draw a conclusion. No FIR data are available for NGC 3489 (not in the plot), which we classify as Compton thick from its $F_{\mathrm{X}} / F_{\text {[OIII] }}$ ratio $(0.63)$.

Objects with an uncertain behaviour constitute a small fraction of the Seyfert 2 sample (7 out of 34, marked with a "?" in Table 2). Most of these objects, placed on the SB region of the diagram, may even not contain an AGN, actually NGC 1058, NGC 3627 and NGC 3486 only have upper limits in X-rays.

In summary, a sub-sample of 11 secure Compton thick candidates have been recognized (they have been marked with a $\sqrt{ }$ in Col. 6 of Table 2); these objects are all Compton thick according to the flux diagnostic diagram and in five cases this indication is supported by a strong Fe line of a $>1 \mathrm{keV}$ equivalent width, i.e. NGC 1068 (Matt et al. 1997), NGC 1667 (Bassani et al. 1999), NGC 2273 (Maiolino et al. 1998), NGC 3079 (Cecil et al. 2002) and NGC 5194 (Terashima \& Wilson 2001) ${ }^{4}$.

These tools have revealed that the fraction of objects which may be affected by Compton thick obscuration among type 2 Seyferts ranges from $\sim 30 \%$ (if only the secure Compton thick sources are considered) up to $\sim 50 \%$ (if also objects with an uncertain behaviour are Compton thick sources) in agreement with previous estimates available for a flux-limited sample (Risaliti et al. 1999) and a distance limited sample (C06).

The X-ray luminosities of Compton thin sources (both type 1 and type 2 Seyferts) have been corrected for the column density measured from the $2-10 \mathrm{keV}$ spectra. Assuming that all the secure candidates are actually Compton thick, then their intrinsic column density should be higher than $\sim 10^{24} \mathrm{~cm}^{-2}$. This prevents any possibility to directly measure the intrinsic X-ray luminosity in the $2-10 \mathrm{keV}$ band, since the latter is completely blocked by the absorbing matter and we can only measure a reflected/scattered component, if any. Therefore, to estimate the intrinsic X-ray luminosity we corrected the observed one by a factor of 60 . This value has been obtained by means of the $F_{2-10 \mathrm{kev}} / F_{\text {[OIII] }}$ ratio: the mean value of this ratio has been calculated for our type 1 objects $\left(\log \left\langle F_{2-10 \mathrm{keV}} / F_{[\mathrm{OIII}]}\right\rangle \sim 1.74 \pm 0.49\right)$ and for the five known Compton thick sources $\left(\log \left\langle F_{2-10 \mathrm{keV}} / F_{[\mathrm{OIII}]}\right\rangle \sim-0.03 \pm 0.07\right)$. The difference between these mean values can be considered as an approximate value of the correction factor when estimating the intrinsic luminosity of our Compton thick sources, i.e.,

\footnotetext{
4 Note that in the other six cases, the non detection of an Fe line could be due to the poor statistics of the X-ray spectra.
} 

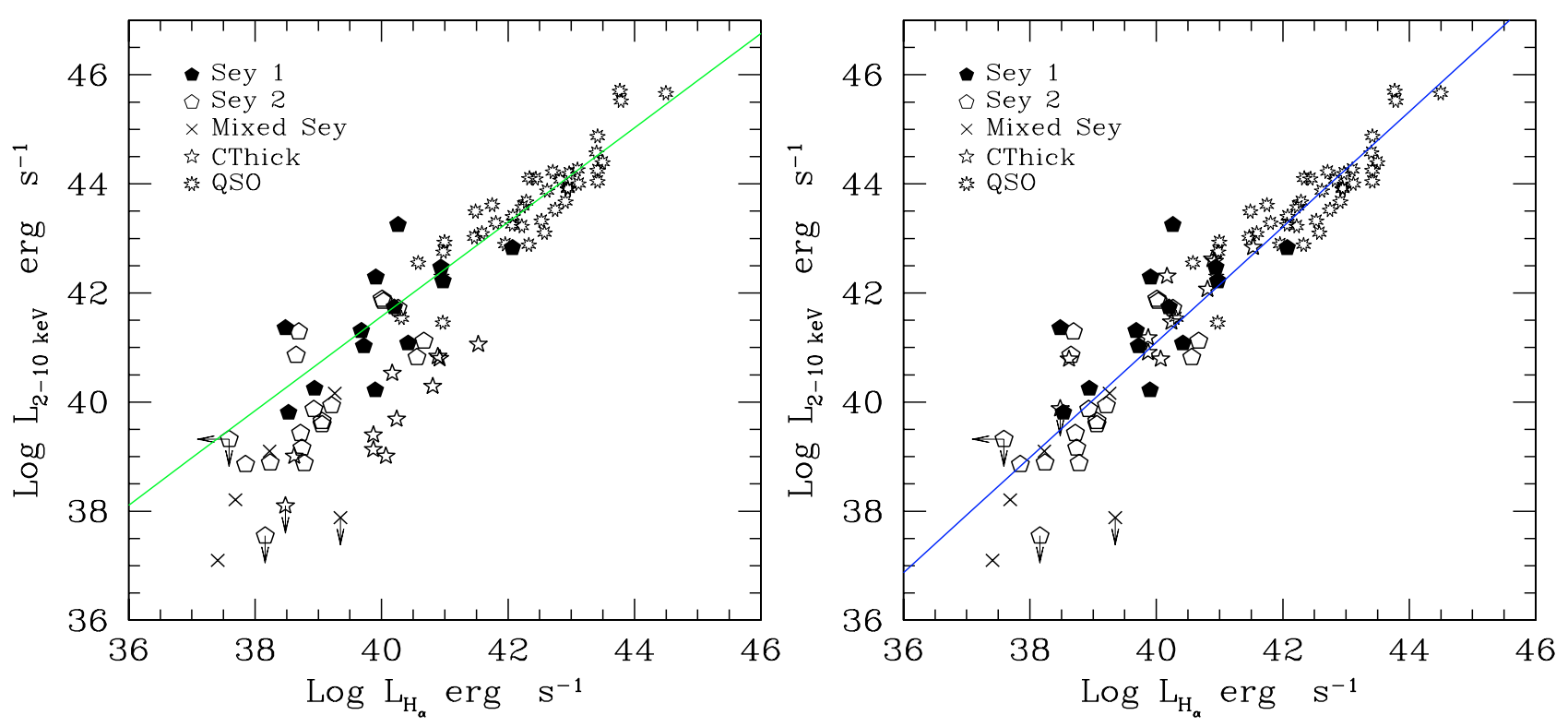

Fig. 2. Left panel: $\log$ of $2-10 \mathrm{keV}$ luminosity versus $\log$ of $\mathrm{H}_{\alpha}$ luminosity (narrow+broad components) corrected for the Galactic and NLR extinction. The dotted line shows the best fit linear regression obtained by fitting type 1 Seyferts and low- $z$ quasars from Ward et al. (1988). Right panel: the same plot using the "Compton thick" corrected luminosity. The solid line shows our best fit linear regression line obtained by fitting the total sample of Seyfert galaxies and the low-z quasars. Filled polygons are type 1 Seyfert, open polygon sare type 2 Seyfert, "mixed Seyfert" objects are indicated as crosses, Compton thick candidates are stars and low- $z$ quasars are "rounded-stars".

$\left\langle F_{2-10 \mathrm{keV}} / F_{[\mathrm{OIII}]}\right\rangle_{\text {Type } 1} /\left\langle F_{2-10 \mathrm{keV}} / F_{[\mathrm{OIII}]}\right\rangle_{\text {Type2,CThick }} \sim 60$, hereafter we refer to "Compton thick" corrected X-ray luminosities (see also C06). In Table 2, X-ray luminosities for the sample sources have been corrected for the intrinsic absorption measured from their X-ray spectra, while for Compton thick candidates we applied the above correction factor. Although the latter should be considered as an indicative value, it is however in tune with what observed with BeppoSAX in Circinus Galaxy (Matt et al. 1999) and NGC 4945 (Guainazzi et al. 2000). Moreover, in the case of NGC 1068, Iwasawa et al. (1997) estimate the intrinsic luminosity to be in the range of $10^{43}-10^{44} \mathrm{erg} / \mathrm{s}$ which is a factor of 100-1000 higher than the observed luminosity. This large correction factor is also theoretically justified by the torus model proposed by Ghisellini et al. (1994), in which they derive, a correction factor ranging from 100 to $2 \times 10^{3}$ depending on the amount of absorption and on the viewing angle with respect to the obscuring torus. Finally, it is worth noticing that before correcting the X-ray luminosity for the Compton thick sources, type 1 and type 2 Seyfert galaxies show a different distribution in luminosities (with KS test of 0.001), type 2 objects have lower luminosities than type 1 objects. After correcting for Compton thick sources, the KS probability is reduced to 0.05 , confirming the previous finding by $\mathrm{C} 06$.

\section{X-ray vs. optical emission line luminosity correlations}

The detection of an X-ray nucleus in almost all our sources is a strong evidence in favour of the presence of an AGN even at very low luminosities. Another convincing way to investigate the activity of the central source is through the observed correlations between X-ray and optical emission line luminosities since a proportionality between these quantities is expected in AGN. In luminous sources strong correlations between $\mathrm{H}_{\alpha}$, $\mathrm{H}_{\beta}$, [OIII $]_{\lambda 5007}$ luminosities and X-ray luminosities have already been found (Elvis et al. 1984; Ward et al. 1988; Mulchaey et al. 1994).
It is worth investigating whether this correlation holds in our sample, in order to further test whether the same physical processes occurring in luminous AGN are also in action in their low luminosity counterparts.

To characterize quantitatively an apparent correlation between two properties of the sample under study we apply a linear regression statistical procedure. We have used the EM (Expectation-Maximization) algorithm since it deals with censored data; this algorithm is implemented in the ASURV package (Isobe et al. 1986).

Table 3 shows the statistical properties of the correlations. The sample has been grouped in sub-classes (type 1 and type 2); quasar samples (as described below) have been added to the analysis.

\subsection{X-ray vs. $H_{\alpha}$ luminosity}

A strong positive correlation between the $\mathrm{X}$-ray and the $\mathrm{H}_{\alpha}$ emission line luminosity has been widely observed in classical AGN, such as Seyferts and quasars (Ward et al. 1988) and in low luminosity AGN, such as LINERs (Terashima et al. 2000; Ho et al. 2001). It has been shown that this correlation is not an artifact of distance effects. Typical ratios observed in bright objects are $\log \left(L_{\mathrm{X}} / L_{\mathrm{H} \alpha}\right) \sim 1-2$, supporting the idea that optical emission lines arise in gas photoionized by the central nucleus.

In Fig. 2 the logarithm of the $2-10 \mathrm{keV}$ luminosity has been plotted versus the $\log L_{\mathrm{H} \alpha}$, the latter including both the narrow and broad (if present) components of the line, corrected for extinction due to the Galaxy and to the narrow-line region (Ho et al. 1997a). The X-ray luminosities are from this work (Table 2) without (left panel) and with (right panel) correction applied to Compton thick candidates. A sample of low- $z$ quasars from Ward et al. (1988) has also been included to compare our results with high luminosity objects (luminosities have been adjusted to $H_{0}=75 \mathrm{~km} \mathrm{~s}^{-1} \mathrm{Mpc}^{-1}$ ). Each class of AGN has been marked with different symbols: filled polygons are type 1 Seyfert, open polygons are type 2 Seyferts, crosses are "mixed Seyferts", stars 
Table 3. Correlation statistics in luminosities.

\begin{tabular}{|c|c|c|c|c|c|c|c|c|}
\hline $\begin{array}{c}\text { Variables } \\
\text { (1) }\end{array}$ & $\begin{array}{l}\text { Sample } \\
\text { (2) }\end{array}$ & $\begin{array}{l}N \\
(3)\end{array}$ & $\begin{array}{c}X(\mathrm{ul}) \\
(4)\end{array}$ & $\begin{array}{c}Y(\mathrm{ul}) \\
(5)\end{array}$ & $\begin{array}{l}\text { rho } \\
(6)\end{array}$ & $\begin{array}{l}\text { Prob. } \\
\text { (7) }\end{array}$ & $\begin{array}{c}a \\
(8)\end{array}$ & $\begin{array}{c}b \\
(9)\end{array}$ \\
\hline $\log L_{\mathrm{X}}$ Vs. $\log L_{\mathrm{H} \alpha}$ & Tot & 47 & 1 & 4 & 0.82 & $<0.001$ & $1.20 \pm 0.12$ & $-6.82 \pm 4.82$ \\
\hline $\log L_{\mathrm{X}}$ vs. $\log L_{\mathrm{H} \alpha}$ & S1 & 13 & 0 & 0 & 0.68 & 0.019 & $0.74 \pm 0.21$ & $12.12 \pm 8.34$ \\
\hline $\log L_{\mathrm{X}}$ vs. $\log L_{\mathrm{H} \alpha}$ & S2 & 34 & 1 & 4 & 0.83 & $<0.001$ & $1.28 \pm 0.14$ & $-10.04 \pm 5.61$ \\
\hline $\log L_{X}$ vs. $\log L_{\mathrm{H} \alpha}$ & $\mathrm{Tot}+\mathrm{QSO}^{a}$ & 87 & 1 & 4 & 0.95 & $<0.001$ & $1.06 \pm 0.04$ & $-1.14 \pm 1.78$ \\
\hline $\log L_{\mathrm{X}}$ vs. $\log L_{\mathrm{H} \alpha}$ & $\mathrm{S} 1+\mathrm{QSO}^{a}$ & 53 & 0 & 0 & 0.93 & $<0.001$ & $0.86 \pm 0.05$ & $6.98 \pm 2.08$ \\
\hline $\log L_{X}$ vs. $\log L_{[\mathrm{OIII}]}$ & Tot & 45 & 1 & 4 & 0.88 & $<0.001$ & $1.22 \pm 0.11$ & $-7.55 \pm 4.33$ \\
\hline $\log L_{\mathrm{X}}$ vs. $\log L_{[\mathrm{OIII}]}$ & S1 & 13 & 0 & 0 & 0.93 & $<0.001$ & $0.86 \pm 0.09$ & $6.99 \pm 3.51$ \\
\hline $\log L_{\mathrm{X}}$ vs. $\log L_{[\mathrm{OIII}]}$ & S2 & 32 & 1 & 4 & 0.86 & $<0.001$ & $1.34 \pm 0.15$ & $-12.32 \pm 5.80$ \\
\hline $\log L_{\mathrm{X}}$ vs. $\log L_{[\mathrm{OIII}]}$ & $\mathrm{Tot}+\mathrm{QSO}^{b}$ & 66 & 1 & 4 & 0.93 & $<0.001$ & $1.22 \pm 0.06$ & $-7.34 \pm 2.53$ \\
\hline $\log L_{X}$ vs. $\log L_{[\mathrm{OIII}]}$ & $\mathrm{S} 1+\mathrm{QSO}^{b}$ & 34 & 0 & 0 & 0.83 & $<0.001$ & $0.95 \pm 0.07$ & $3.87 \pm 2.76$ \\
\hline
\end{tabular}

Notes: Statistical properties of the "Compton thick" corrected 2-10 keV X-ray luminosity versus $\mathrm{H}_{\alpha}$ and [OIII] $\lambda 5007$ luminosities; Col. (1): variables $X$ and $Y$; Col. (2): Subsample considered: Tot $=$ total Seyfert sample, S1 = Seyfert 1 galaxies, S2 = Seyfert 2 galaxies; QSO ${ }^{a}=$ low- $z$ quasars (Ward et al. 1988); $\mathrm{QSO}^{b}=$ bright type 1 Seyfert (Mulchaey et al 1994) + PG quasars (Alonso-Herrero et al. 1997); Col. (3): number of objects; Cols. (4) and (5): Number of upper limits in variable $X$ and $Y$; Cols. (6) and (7) Spearman's rho correlation coefficient and the associated probability $\mathrm{P}$ for accepting the null hypothesis that there is no correlation; Cols. (8) and (9): correlation coefficient of the best fit linear regression line calculated using EM algorithm (Isobe et al. 1986), $Y=a \times X+b$.

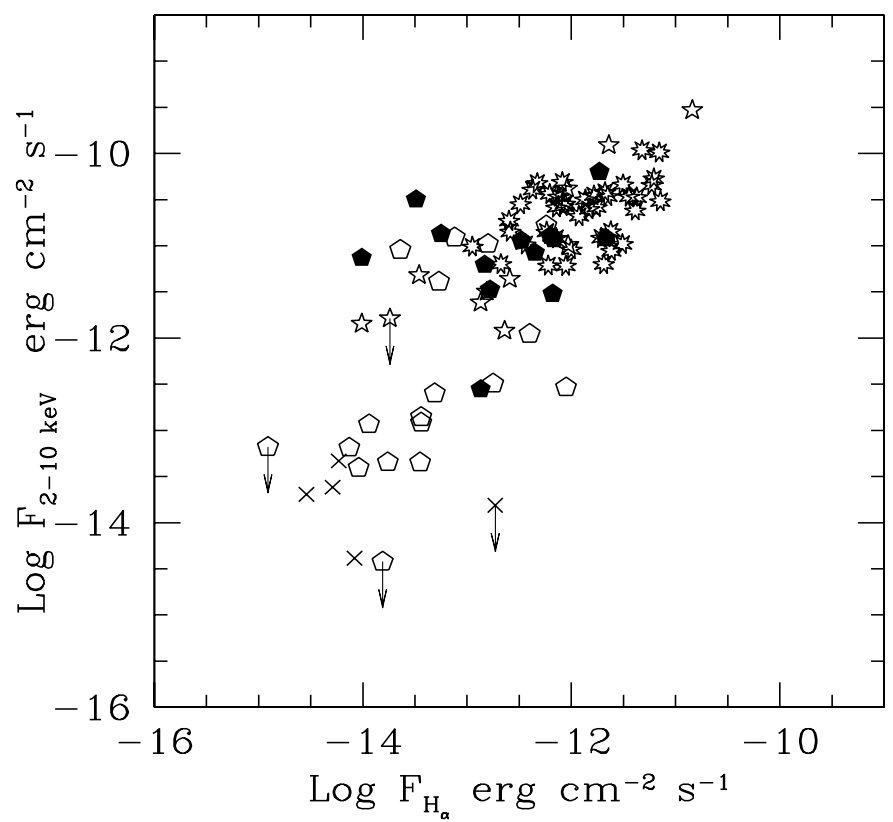

Fig. 3. Correlation between $2-10 \mathrm{keV}$ versus $\mathrm{H}_{\alpha}$ fluxes. $\mathrm{H}_{\alpha}$ narrow+broad components are corrected for the Galactic and NLR extinction. Symbols as in Fig. 2.

are the Compton thick candidates and rounded-stars are low- $z$ quasar objects.

First, we checked whether a correlation is present among our Seyfert sample. As reported in Table 3, a strong correlation is found in the sample. We fitted type 1 Seyferts and low- $z$ quasar objects in order to compare objects with broad+narrow component of the $\mathrm{H}_{\alpha}$ line. The solid line in the left panel represents our best-fit linear regression line. This correlation is highly significant (Spearman's rho $=0.93$ ), although type 1 Seyferts in our sample show a larger scatter than the low-z quasars. Note that the X-ray and $\mathrm{H}_{\alpha}$ data have not been taken simultaneously. Consequently, the strong X-ray flux variability observed in most type 1 Seyferts (NGC 3516, Guainazzi et al. 2001; NGC 4051,
Lamer et al. 2003; NGC 4151, Czerny et al. 2003, NGC 4395, Iwasawa et al. 2000;) is a likely source of scatter. At lower luminosities, type 2 objects and "mixed Seyferts" show $L_{\mathrm{X}} / L_{\mathrm{H} \alpha}$ ratios lower than type $1 \mathrm{~s}$ and low- $z$ quasars. Note, however, that we are comparing type 1 objects which have both narrow and broad $\mathrm{H}_{\alpha}$ line components and type 2 Seyferts which lack the broad $\mathrm{H}_{\alpha}$ component.

On the right panel of Fig. 2, the 2-10 keV luminosity of Compton thick candidates has been corrected and the solid line is our best-fit regression line obtained by fitting the total Seyfert sample and the low- $z$ quasar sample. This regression line is consistent with that found by Ho et al. (2001) using the same low- $z$ quasar sample and a sample of LLAGN observed with Chandra.

The correction in the X-ray luminosity significantly reduces the scatter at low luminosities and the inclusion of type 2 Seyferts in the calculation of the regression line results in a steeper slope with respect to those of type 1 and low- $z$ quasars. The steepening of the regression line is probably enhanced by those Compton thin type 2 objects at low luminosities, which show an optical excess emission with respect to the X-ray luminosity. The latter could be due to the contribution given by a circumnuclear starburst to the $\mathrm{H} \alpha$ emission which is more important in low luminosity sources than in high luminosity ones, where the emission is completely dominated by the AGN.

The same correlation using fluxes is shown in Fig. 3 (rho = 0.78 , prob $<0.001$ ) strongly supporting that the luminosityluminosity correlation is not due to distance effects.

It is remarkable that the present sample traces the $L_{\mathrm{X}}-L_{\mathrm{H} \alpha}$ relation for luminosities typical of luminous AGN $\left(10^{41}-10^{43} \mathrm{erg} \mathrm{s}^{-1}\right)$ down to low luminosities typical of LINERs $\left(10^{37}-10^{40} \mathrm{erg} \mathrm{s}^{-1}\right)$. The strong correlation observed between $L_{\mathrm{X}}-L_{\mathrm{H} \alpha}$ suggests that the dominant ionization source in our sample is photoionization by an AGN and that optical/UV photons are somehow linked to the X-ray emission. A few objects at $2-10 \mathrm{keV}$ luminosities of $\sim 10^{38} \mathrm{erg} \mathrm{s}^{-1}$ appear to be systematically under the correlation. Three out of four of these sources are actually those classified as "mixed Seyfert", i.e. NGC 2683, NGC 3627 and NGC 6503; moreover for NGC 1058 and NGC 3627 only an upper limit to the X-ray luminosity is 

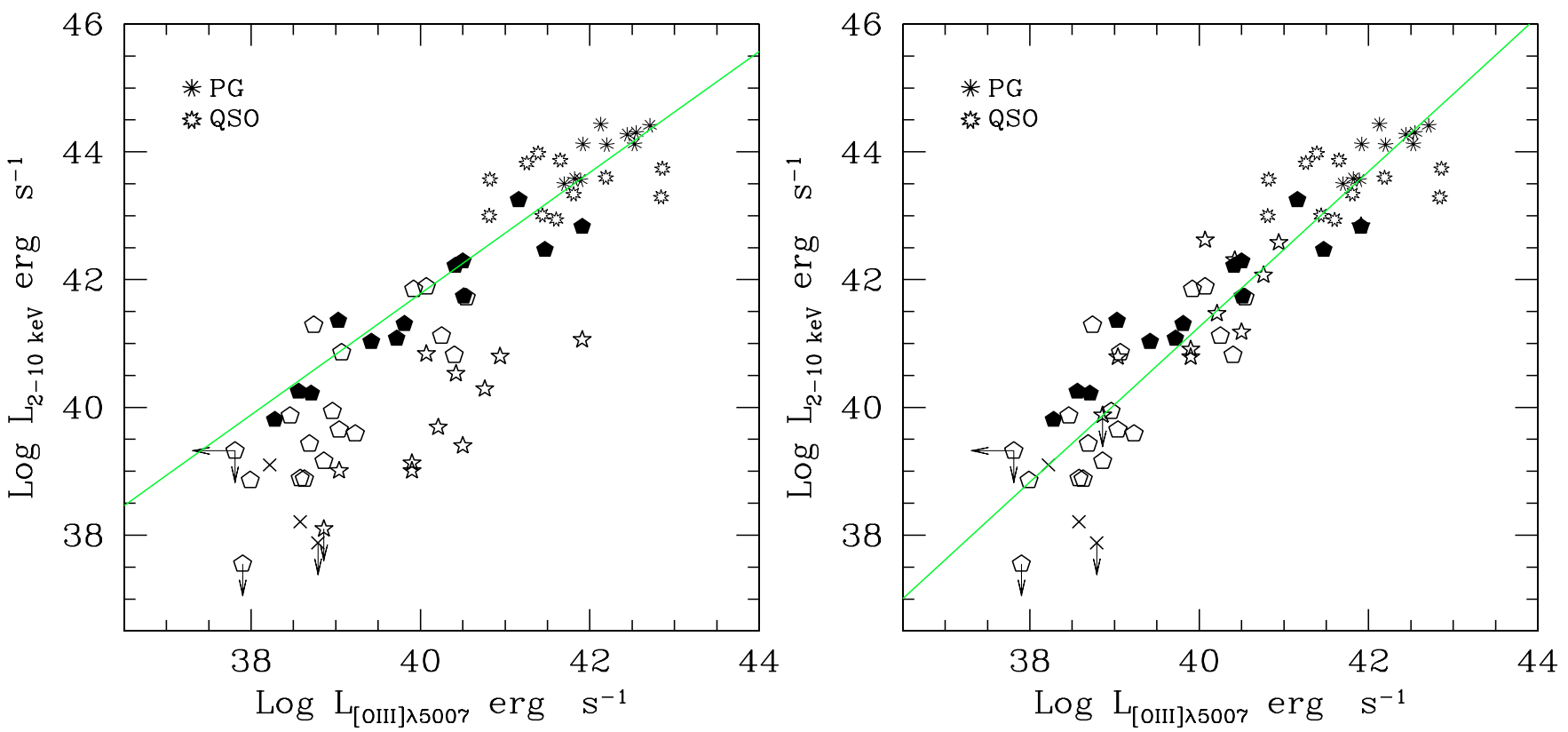

Fig. 4. Left panel: $\log$ of 2-10 keV luminosity versus $\log$ of $[\mathrm{OIII}]_{25007}$ luminosity corrected for the Galactic and NLR extinction. The solid line shows the best fit linear regression line obtained by fitting type 1 Seyfert, a sample of bright type 1 Seyferts (QSO, Mulchaey et al. 1994) and a sample of PG quasar (PG, Alonso-Herrero et al. 1997). Right panel: the same plot using "Compton thick" corrected luminosity. The solid line shows our best fit linear regression line for the total sample of Seyfert galaxies, the PG quasars and bright type 1 Seyfert. Symbols are as in Fig. 2.

available. If the ionization mechanism of the emission lines is due to or enhanced by hot stars or shocks, the resulting $L_{\mathrm{X}} / L_{\mathrm{H} \alpha}$ ratio is expected to be accordingly smaller compared to those of AGN. Indeed, Pérez-Olea \& Colina (1998) have shown how the $L_{\mathrm{X}} / L_{\mathrm{H} \alpha}$ ratio in AGN is larger than in starbursts up to a factor of $\sim 100$ independently on the intrinsic luminosity or activity level. This could be a possible scenario for these sources which, from our diagnostic diagrams, have been recognized as our most probable starburst candidates. However we cannot rule out that a very faint/heavily obscured AGN is present in such objects, as for example in the "Elusive AGN" in which the nucleus is heavily obscured and there are no optical evidences for the presence of an AGN (Maiolino et al. 2003).

\subsection{X-ray vs. $[\mathrm{OIII}]_{\lambda 5007}$ luminosity}

As previously discussed in Sect. 4 , the $[\mathrm{OIII}]_{\lambda 5007}$ emission line is thought to be an isotropic indicator and should, therefore, be representative of the intrinsic power of the central engine. Although the properties of $[\mathrm{OIII}]_{\lambda 5007}$ have been widely investigated in the literature, the $L_{\mathrm{X}}$ vs. $L_{\text {[OIII] }}$ correlation itself has been poorly studied. Mulchaey et al. (1994) have found a strong correlation between the emission line flux and the observed UV continuum in type 1 Seyferts, while no correlation was observed in type 2 Seyferts. This finding is in agreement with the torus model, where the UV photons are scattered while the line emission is produced by a directly viewed component. However these authors made use of an heterogeneous sample and did not take into account the presence of Compton thick sources. More recently, Heckman et al. (2005) have found that $L_{X}$ and $L_{[\mathrm{OIII}}$ are well correlated in a sample of hard X-ray selected AGN (both type 1 and type 2). They also found that for a sample of [OIII] flux selected sources, the correlation $L_{\mathrm{X}}$ vs. $L_{[\mathrm{OIII}]}$ for type $1 \mathrm{ob}-$ jects is consistent with that found in the type 1 hard X-ray sample. However, the type 2 objects spread a much wider range in

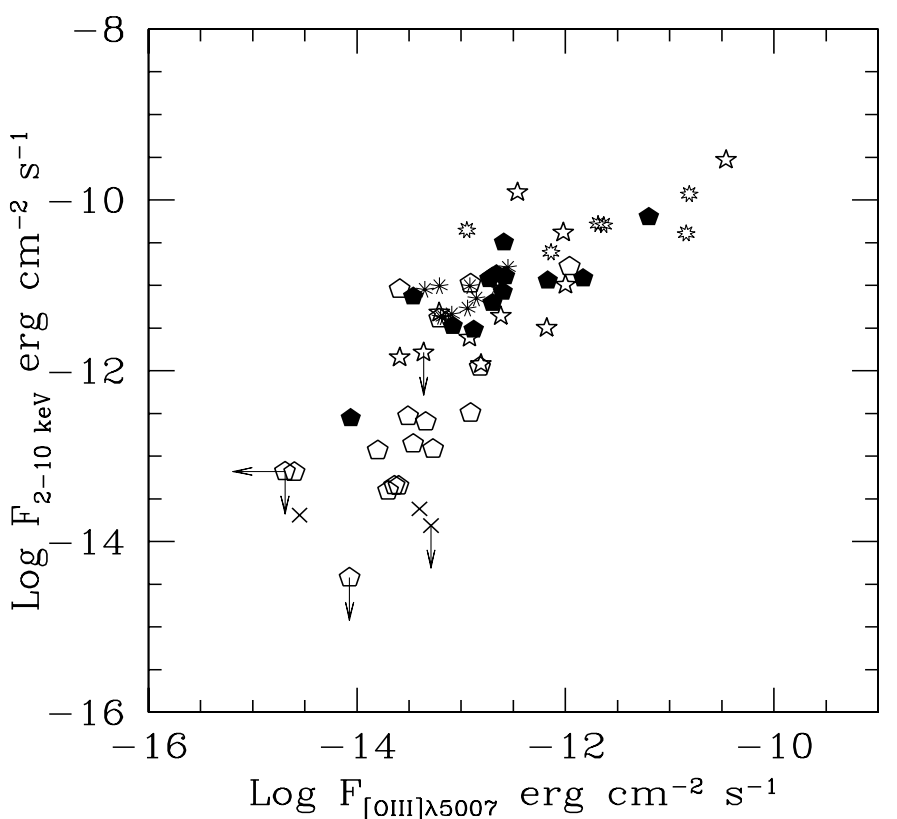

Fig. 5. Correlation between $\log$ of $2-10 \mathrm{keV}$ versus $\log$ of $[\mathrm{OIII}]_{\lambda 5007}$ fluxes, the latter corrected for the Galactic and NLR extinction.

the luminosity ratio and many of them are very weak in hard X-rays.

In Fig. 4 we show the $2-10 \mathrm{keV}$ luminosity (without correction for Compton thick sources, left panel, and with correction, right panel) versus the $[\mathrm{OIII}]_{25007}$ luminosity (corrected for the Galactic and NLR extinction, Ho et al. 1997a). Two comparison samples of bright AGN have been included in the analysis chosen for having both X-rays and $[\mathrm{OIII}]_{\lambda 5007}$ fluxes available: 1) a sample of luminous type 1 Seyfert galaxies (hereafter QSO) from Mulchaey et al. (1994); 2) a sample of PG quasars (hereafter PG) from Alonso-Herrero et al. (1997). Luminosities 
have been adjusted to $H_{0}=75 \mathrm{~km} \mathrm{~s}^{-1} \mathrm{Mpc}^{-1}$. The two chosen samples of luminous AGN are not meant to be complete and biases against low luminosity objects are probably introduced. However the low luminosity ranges are covered by our sample and they are just taken as representatives of the class of luminous sources.

The solid line in the left panel of Fig. 4 is the best fit linear regression line obtained by fitting type 1 Seyferts, the QSO sample and the PG sample. Contrary to what is obtained in the $L_{\mathrm{X}}$ vs. $L_{\mathrm{H} \alpha}$ relation, most of the scatter here is introduced by QSOs, while type 1 Seyferts of our sample follow a tighter correlation. There is a clear separation between type 1 and type 2 Seyferts. Once we correct the X-ray luminosity assuming the presence of "Compton thick" sources, the correlation between the two luminosities is tighter as shown in the right panel of Fig. 4 in which the best fit linear regression line for the total sample, the QSO and PG samples is over-plotted. As also found in the $L_{X}$ vs. $L_{\mathrm{H} \alpha}$ correlation, the slope of the regression line is steeper with respect to those obtained for only type 1 and low-z quasars. At low luminosities, a possible contribution by a circumnuclear starburst to the [OIII] emission could explain the excess of the [OIII] luminosity with respect to the X-ray one observed in a few sources. Actually, at very low luminosities, the same sources which lay below the correlation in the X-ray vs. $\mathrm{H}_{\alpha}$ plot, lay also below the $L_{\mathrm{X}}$ vs. $L_{[\mathrm{OIII}]}$ relation (e.g., NGC 1058, NGC 2683 and NGC 3627). The X-ray versus [OIII] correlation still holds in the flux-flux plot of Fig. 5 (rho $=0.78$, Prob < 0.001). As for the $\mathrm{H}_{\alpha}$ luminosity, also the $[\mathrm{OIII}]_{\lambda 5007}$ flux appears to be a good tracer of the AGN power, and both correlations are good tools to estimate the expected X-ray luminosity. The following relations are obtained for our sample:

$\log L_{\mathrm{X}}=(1.06 \pm 0.04) \log L_{\mathrm{H} \alpha}+(-1.14 \pm 1.78)$

$\log L_{\mathrm{X}}=(1.22 \pm 0.06) \log L_{[\mathrm{OIII}]}+(-7.34 \pm 2.53)$.

\section{6. $M_{\mathrm{BH}}$ and Eddington ratios}

Black hole mass $\left(M_{\mathrm{BH}}\right)$ estimates are available for 44 out of 47 objects in our sample (Col. 9, Table 2). The $M_{\mathrm{BH}}$ from the literature, have been estimated in different ways from gas, stellar and maser kinematics to reverberation mapping or inferred from the mass-velocity dispersion correlations (Ferrarese 2002; Tremaine et al. 2002). For a group of objects stellar velocity dispersions are available from di Nella et al. (1995), Barth et al. (2002) and McElroy (1995), and we calculate the $M_{\mathrm{BH}}$ using the Tremaine et al. (2002) relation. In Table 2 we report all these $M_{\mathrm{BH}}$ estimates, the method used to calculate them and the corresponding reference.

As shown in Fig. 6 (left panel), black hole masses are fairly sampled from $\sim 10^{5}$ to $10^{8} M_{\odot}$ with a peak at $10^{7-8} M_{\odot}$; this figure further indicates that type 1 and type 2 Seyferts show a similar distribution. Woo \& Urry (2002) presented a large compilation of black hole masses, for an heterogeneous sample of 234 AGN, ranging from $\sim 10^{6}$ to $\sim 10^{10} M_{\odot}$. From a comparison of different methods in estimating $M_{\mathrm{BH}}$, they found that $M_{\mathrm{BH}}$ values estimated from reverberation mapping and stellar velocity dispersion are those more reliable. Uncertainties in $M_{\mathrm{BH}}$ estimates for our sample are probably introduced by the scatter in the mass-velocity dispersion correlations and are typically of the order of $0.3-0.5$ dex. We refer to the above mentioned papers for a detailed description of the different methods used to estimate $M_{\mathrm{BH}}$ and the relative errors associated to these measurements.

The $L_{\mathrm{Bol}} / L_{\mathrm{Edd}}$ ratio distribution is plotted in the right panel of Fig. 6. To calculate the bolometric luminosity we have assumed that $L_{\mathrm{Bol}} / L_{2-10 \mathrm{keV}} \sim 30$. The latter value is typical of luminous AGN, being normally in the range of 25-30 (Risaliti \& Elvis 2004, Elvis et al. 1994). However, it must be kept in mind that the bolometric luminosity here is just a multiple of the X-ray luminosity, while it really depends on the shape of the spectral energy distribution which could differ among high and low luminosity AGN (Ho 1999; Marconi et al. 2004); actually, in LLAGN, the observed $L_{\mathrm{Bol}} / L_{2-10 \mathrm{keV}}$ ratio ranges from 3 to 16 (Ho 1999). The $L_{\mathrm{Bol}} / L_{\mathrm{Edd}}$ ratio distribution for the total Seyfert sample covers a wide range of Eddington ratios going down to $10^{-7}$. If a $L_{\mathrm{Bol}} / L_{2-10 \mathrm{kev}}$ ratio of 10 is considered, then Eddington ratios would be a factor of 3 lower than those in Fig. 6. The distribution of type 1 objects has been marked with a shaded region. It is interesting to note that a Kolmogorov-Smirnov test gives a probability of 0.01 for the type 1 and type 2 sub-samples of being drawn from the same parent population, suggesting that type 2 objects are accreting at lower Eddington ratios with respect to type 1 Seyfert galaxies. However, there are some caveats to take into account: i. the KS probability of the type 1 and type 2 $\mathrm{X}$-ray luminosity distribution is 0.05 , consequently affecting the bolometric luminosity distributions; ii. as already pointed out, the bolometric correction could be not a constant, depending for example on luminosity and therefore could change from object to object; iii. objects with an uncertain behaviour, those in which the star-formation probably dominates, have been included in the type 2 class. As a matter of fact, if "mixed Seyferts" are excluded from the type 2 sub-sample, the KS probability of the $L_{\mathrm{Bol}} / L_{\mathrm{Edd}}$ ratio distributions is 0.05 . Moreover, it is possible that with the present data we are not able to detect all the absorbed low luminosity type 2 s, e.g. Compton thick sources not recognized to be such by the diagnostic tools and/or objects with part of the X-ray emission absorbed by parsec scale clumpy material detected in a low $N_{\mathrm{H}}$ state (e.g., NGC 4388, Elvis et al. 2004). However, if a trend of type 2 Seyfert galaxies having lower Eddington ratios than type 1 ones is present, this would have several interesting implications: for example it would nicely confirm a model proposed by Nicastro (2000) that relates the formation of the broad emission lines of active galactic nuclei to the accretion rates, i.e. for very low accretion rates the BLR would no longer exist. More data are needed to have a complete sample and statistically confirm these findings.

In Fig. 7, Compton thick corrected X-ray luminosities have been plotted against black hole mass. No correlation is found between these two parameters. The same considerations can be applied when $\mathrm{H}_{\alpha}$ and [OIII] luminosities are plotted against $M_{\mathrm{BH}}$, i.e. no correlation with $M_{\mathrm{BH}}$ is observed, which is an expected result given the correlation of these quantities with the X-ray luminosity. Some previous studies found a correlation between the AGN luminosity and $M_{\mathrm{BH}}$ (Koratkar \& Gaskell 1991; Kaspi et al. 2000), however our result is in agreement with that found by Pellegrini (2005) for a sample of SMBH in the local universe for which Chandra nuclear luminosities were available. Also Woo \& Urry (2002) found no correlation between bolometric luminosity and black hole masses. Interestingly, neither is radio emission correlated to black hole mass in nearby nuclei (Ho 2002).

We have over-plotted $L_{\mathrm{X}}$ as a function of $M_{\mathrm{BH}}$ for Eddington ratios of $1.0,0.01$ and $10^{-4}$ (solid lines in Fig. 7). Woo \& Urry (2002) have shown that bright local AGN normally show Eddington ratios which span three orders of magnitude down to $L_{\mathrm{Bol}} / L_{\mathrm{Edd}} \sim 10^{-3}$, while at higher redshifts the Eddington ratios distribution is narrower, i.e. $L_{\mathrm{Bol}} / L_{\mathrm{Edd}}$ peaks at around $1 / 3$ with a dispersion of $0.3 \mathrm{dex} \mathrm{rms}$, as recently shown by Kollmeier et al. (2005) for a sample of AGN discovered in the AGES Survey 

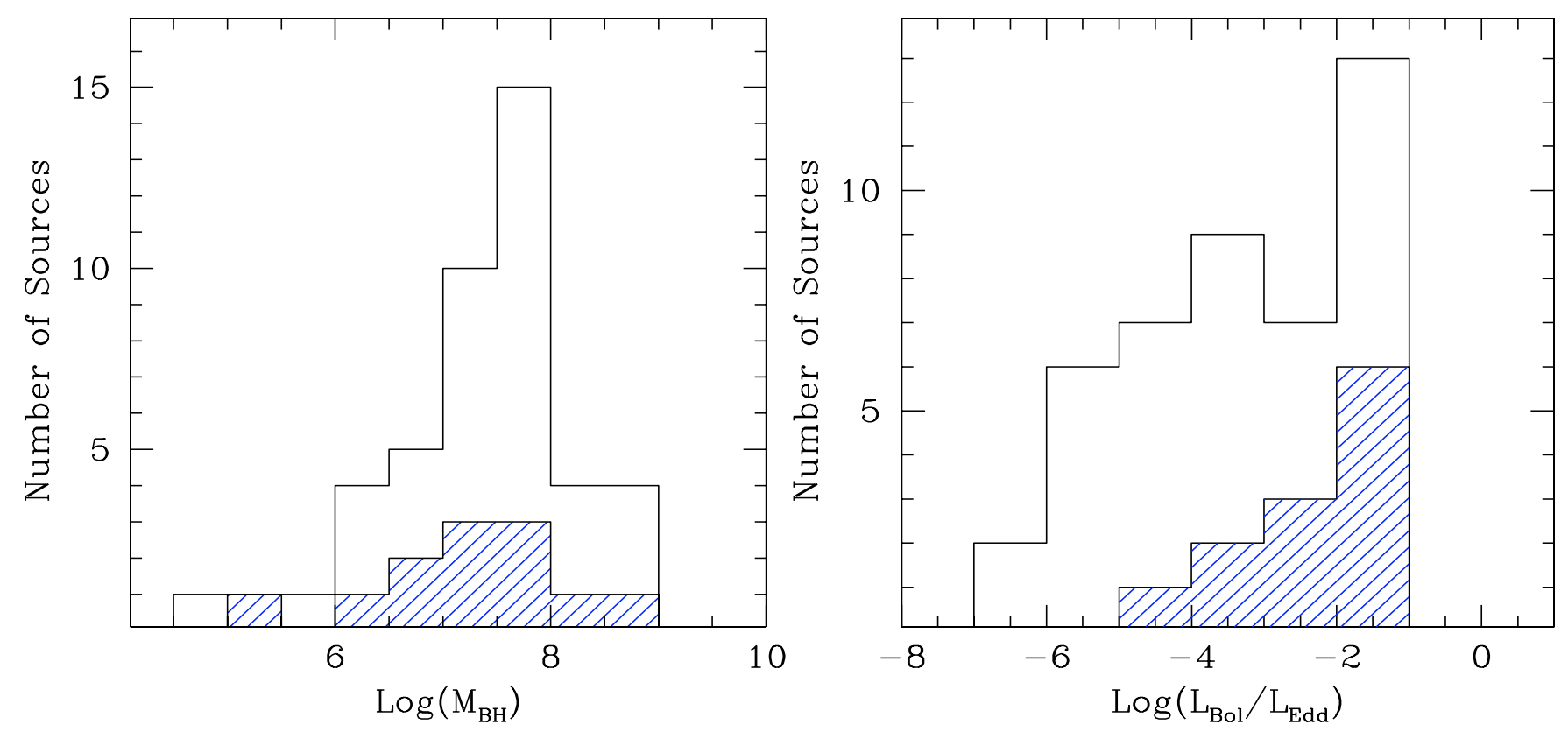

Fig. 6. Left panel: distribution of the log of black hole masses in unit of solar masses. Right panel: distribution of the $\log$ of $L_{\mathrm{Bol}} / L_{\mathrm{Edd}}$ ratio, assuming that $L_{\mathrm{Bol}} / L_{\mathrm{X}} \sim 30$. In both panels the shaded areas represent the distribution of type 1 Seyferts only.

covering a redshift range of $0.3-4$. Indeed, most of our sources are radiating at very low Eddington ratios if compared with luminous AGN. The low Eddington ratios observed in our sample are even lower if the bolometric correction considered is that of LLAGN. At such low Eddington ratios, radiatively inefficient accretion is normally invoked as the putative mechanism for the production of the observed emission. For example, in Merloni et al. (2003) the $L_{2-10 \mathrm{keV}} / L_{\text {Edd }}$ ratio for an heterogeneous sample of AGN and stellar masses black holes ranges from 1 to $10^{-8}$. These authors delineate a range of $L_{2-10 \mathrm{keV}} / L_{\text {Edd }}$ ratios in which the accretion mode changes from a radiatively efficient to a radiatively inefficient one below $10^{-3}$ and above $\sim 0.7$. According to these claims, most of our sources would be powered by radiatively inefficient accretion; this issue is further discussed in Sect. 7. Finally, note that in Fig. 7 Compton thick sources populate the upper part of the plot, at higher Eddington ratios, while only one source has $L_{\mathrm{Bol}} / L_{\mathrm{Edd}} \lesssim 10^{-3}$; this is probably a selection effect, since Compton thick sources with an observed $L_{2-10 \mathrm{keV}} \lesssim$ $10^{38} \mathrm{erg} \mathrm{s}^{-1}$ would probably be undetectable in our sample.

\section{Discussion}

The X-ray analysis performed on a sample of nearby Seyfert galaxies has revealed the presence of a central active nucleus in all but four sources out of 47 . The intrinsic nuclear X-ray luminosities span nearly five orders of magnitude, down to $10^{38} \mathrm{erg} / \mathrm{s}$ in the $2-10 \mathrm{keV}$ energy range. After taking into account the presence of Compton thick objects in which the nuclear emission is heavily reduced by absorbing material, we have found a strong correlation between X-ray and optical line emission luminosities. This suggests a link between the X-ray emission and the ionization radiation which holds also at very low luminosities. In luminous AGN, the UV/optical radiation has the form of a "big blue bump" feature (Elvis et al. 1994), commonly modelled by a geometrically thin accretion disk working in a radiatively efficient regime (Shakura \& Sunyaev 1973) while the X-ray emission is produced by Comptonization of soft photons in a hot corona (Haardt \& Maraschi 1991).

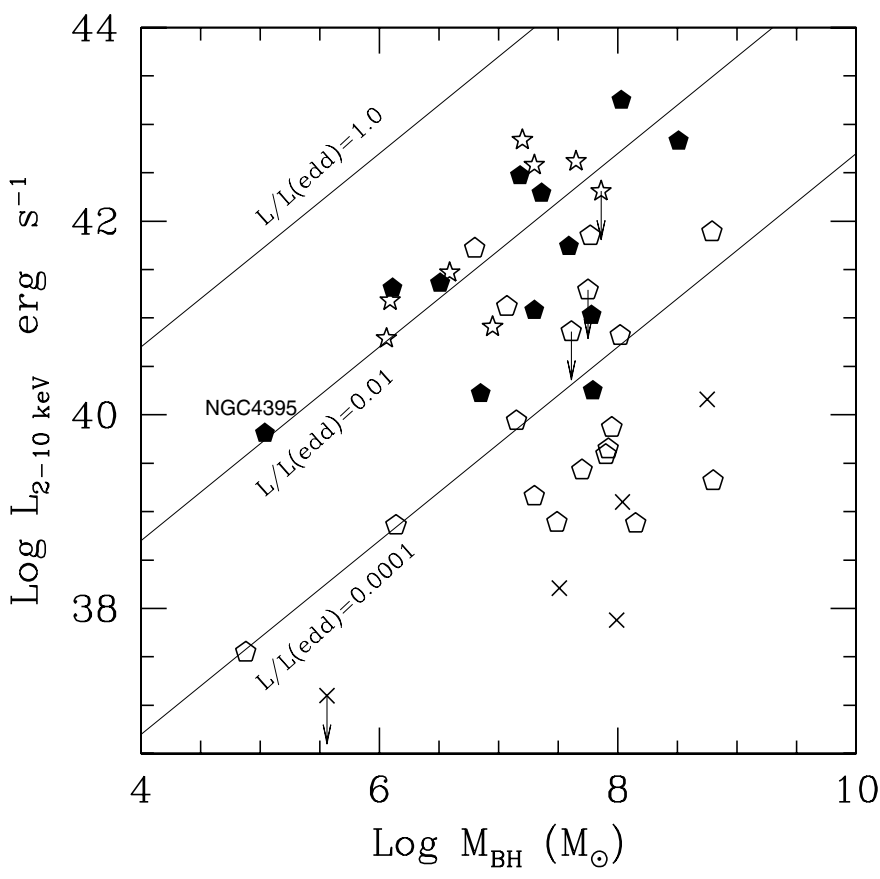

Fig. 7. $\log$ of 2-10 keV luminosity versus $\log$ of black hole mass (symbols as in Fig. 4). Compton thick candidates are plotted as stars. The solid lines show the $L_{2-10 \mathrm{keV}}$ as a function of the black hole mass for Eddington ratios of 1.0 and 0.01 .

On the other hand, it has been shown that some LLAGN lack this "big blue bump" feature (Ho 1999). Such observational evidence combined with the low Eddington ratios commonly observed in LLAGN, has lead many authors to invoke advectiondominated accretion flow models (ADAF, Narayan \& Yi 1994) and their variants to model their spectral shape. As a matter of fact, ADAF models work in a radiatively inefficient regime at sub-Eddington ratios $\left(L<0.01-0.1 L_{\text {Edd }}\right)$ and can reproduce the lack of UV excess observed in the SED of LLAGN. However, also radiatively efficient standard accretion disks are stable at 
low Eddington ratios down to $L \sim 10^{-6} L_{\text {Edd }}$ (Park \& Ostriker 2001) and can reproduce the shape of the LLAGN SED since the temperatures of a multi-colour disk scale with $\dot{m}^{1 / 4}$ (Ptak et al. 2004).

Several examples of sources studied in the literature have shown that reconciling the multi-waveband observations of LLAGN with theoretical models is a complex task. For example, Ptak et al. (2004) have shown that the UV/X-ray spectrum of NGC 3998 could be reproduced equally well by radiatively inefficient accretion flow (RIAF) models and Comptonized thin disk models with $\dot{m}<10^{-5}$ at which a standard disk is both stable and thick. In the cases of M 81 and NGC 4579, two well known LLAGN belonging also to our sample, a geometrically thin disk extending up to $\sim 100$ Schwarzchild radii was required to account for their optical/UV spectra, while the X-ray radiation has been explained by an optically thin, two temperature ADAF at smaller radii (Quataert et al. 1999). NGC 4258 has been considered as an ideal candidate for ADAF model (Lasota et al. 1996), while Yuan et al. (2002) have shown that the jet component dominates the emission. Based on the IR/X-ray Eddington ratio, the $\mathrm{X}$-ray variability and spectral shape, Fiore et al. (2001b) considered NGC 4258 an AGN in a low state or a scaled-down version of a Seyfert galaxy.

As reported in this work, the X-ray versus optical emission line correlations scale with luminosity, so that low luminosity Seyfert galaxies appear to be a scaled-down version of classical AGN. The observed optical emission line spectra suggest that the amount of UV radiation produced is sufficient to ionize the NLR. However, if Seyfert galaxies with very low luminosities in our sample actually lack a "big blue bump" feature, as sometimes observed in LLAGN, then an additional source of UV photons must be invoked which means that the AGN UV photons are not the only contributors to the ionizing radiation, i.e. radiation produced in circumnuclear starburst could contribute significantly to the observed emission line fluxes. A similar scenario has been proposed by Contini (2004) for NGC 4579, in which both line and continuum emission are explained by a composite model which accounts for the presence of an AGN, a jet which interacts with the ISM creating shocks, a starburst of different ages and HII regions. Moreover, some authors have shown that the observed emission line ratios in AGN may be produced by both a thermal or a non-thermal optical/UV continuum (Keel \& Miller 1983; Martins et al. 2003). Indeed, to constrain the spectral shape of these sources and compare it with theoretical models, it is necessary to properly subtract UV/optical data for the Galactic emission and have multi-waveband observations.

\subsection{On the effects of the incompleteness of the sample}

The Palomar sample is one of the best samples available for the kind of study presented here. In Ho \& Ulvestad (2001) a detailed discussion of biases is presented and the sample is compared with other Seyfert samples available in the literature. In this work, only 47 out of 60 Seyfert galaxies belonging to the original complete sample (HFS97) have X-ray data, and this fact certainly introduces incompleteness problems and biases in our study. To investigate this issue, we have looked at the $\mathrm{H}_{\alpha}$ luminosities of the 13 objects without X-ray data and found that they range from $38.43<\log L_{\mathrm{H} \alpha}<40.54 \mathrm{erg} \mathrm{s}^{-1}$ (HFS97). In this range of luminosities we have shown that the X-ray vs. optical emission line correlations still hold: therefore we should expect these sources to have low X-ray luminosities. Of course, given the large scatter observed at low luminosity, a determination of the X-ray luminosity for the total sample is important both to better calibrate the correlations down to low luminosities and also to strengthen our conclusions on the basis of a complete sample. Regarding the analysis involving the black hole mass, the fact that no dependence between luminosity and $M_{\mathrm{BH}}$ has been found, suggests that no significant differences in the total $M_{\mathrm{BH}}$ distribution is expected, if we were able to add the $M_{\mathrm{BH}}$ estimates for these 13 missing sources. Of course, the availability of $M_{\mathrm{BH}}$ estimates for the complete sample of Palomar Seyfert galaxies will reduce some bias effects, albeit, since $M_{\mathrm{BH}}$ estimates have been obtained by using different methods having different degrees of uncertainty, it is intrinsically difficult to estimate the degree of incompleteness of the $M_{\mathrm{BH}}$ data.

\section{Conclusions}

The X-ray analysis of a sample of 47 nearby Seyfert galaxies (type 1, type 2 and "mixed Seyfert") has allowed us to obtain nuclear 2-10 keV X-ray luminosities with minimal contamination by off-nuclear sources and diffuse emission. The dichotomy often observed in the luminosity of type 1 and type 2 AGN, is mainly due to the presence of heavy absorption in type 2 objects, as demonstrated in $\mathrm{C} 06$ and confirmed here. A sub-sample of 11 candidate Compton thick sources in our sample $(>30 \%$ of type 2 Seyferts) has been found. Their observed luminosities have been increased by an indicative factor of 60 to take into account the luminosity obscured from our line of sight. In the effort of further verifying the physical continuity between our sample and bright AGN, the X-ray luminosities, non-corrected and corrected for Compton thick sources, have been correlated with the $\mathrm{H}_{\alpha}$ and the [OIII] luminosities, both suspected to be absorption independent quantities and good tracers of the nuclear emission. X-ray luminosities have also been correlated with $M_{\mathrm{BH}}$. The results obtained are:

- Both $L_{\mathrm{X}}$ vs. $L_{\mathrm{H} \alpha}$ and $L_{\mathrm{X}}$ vs. $L_{[\mathrm{OIII}]}$ correlations are highly significant in our sample, indicating that the $\mathrm{X}$-ray emission and the UV ionizing radiation are linked.

- Both correlations scale with luminosity, i.e. they have similar slopes of more powerful objects, suggesting that low luminosity Seyfert galaxies are powered by the same physical processes which operate in brighter AGN such as QSOs.

- No correlation is found between nuclear luminosity and $M_{\mathrm{BH}}$ in agreement with some previous studies (Woo \& Urry 2002; Pellegrini et al. 2005).

- $L / L_{\text {Edd }}$ ratios span three order of magnitudes down to $L_{\mathrm{Bol}} / L_{\mathrm{Edd}} \sim 10^{-7}$, indicating that most of our sources are accreting at very low Eddington ratios.

Overall our results suggest that Seyfert nuclei in our sample are consistent with being a scaled-down version of luminous AGN, except for a small fraction of objects having luminosities in the $10^{37}-10^{38} \mathrm{erg} \mathrm{s}^{-1}$ range and which show evidences of stellar processes as the underlying agent responsible for the activity. A forthcoming paper is in preparation in which the multiwaveband analysis for this sample of Seyfert galaxies will be enlarged, including the study of nuclear radio, IR and optical emission jointly with the X-ray luminosity and $M_{\mathrm{BH}}$ estimates, and tested against theoretical models.

Acknowledgements. We thank Silvia Pellegrini and Andy Fabian for helpful suggestions. We thank our referee for valuable suggestions. F.P. acknowledges support by a "Juan de la Cierva" fellowship. Financial support for F.P., X.B. and F.J.C. was provided by the Spanish Ministry of Education and Science, under project ESP2003-00812. 


\section{References}

Abramowicz, M. A. 1997, Accretion Disks - New Aspects, LNP 487, 320

Alonso-Herrero, A., Ward, M. J., \& Kotilainen, J. K. 1997, MNRAS, 288, 977

Alonso-Herrero, A., Rieke, M. J., Rieke, G. H., \& Shields, J. C. 2000, ApJ, 530, 688

Antonucci, R.J., \& Miller, J. S. 1985, ApJ 297, 621

Bassani L., Dadina M., Maiolino R., et al. 1999, ApJS 121, 473

Barcons, X., Carrera, F. J., \& Ceballos, M. T. 2003, MNRAS, 339, 757

Barth, A. J., Reichert, G. A., Filippenko, A. V., et al. 1996, AJ, 112, 1829

Barth, A. J., Ho, L. C., Filippenko, A. V., Rix, H., \& Sargent, W. L. W. 2001, ApJ, 546, 205

Barth, A. J., Ho, L. C., \& Sargent, W. L. W. 2002, AJ, 124, 2607

Caccianiga, A., Severgnini, P., Braito, V., et al. 2004, A\&A, 416, 901

Cappi, M., Panessa, F., Bassani, L., et al. 2006, A\&A, 446, 459

Cecil, G., Bland-Hawthorn, J., \& Veilleux, S. 2002, ApJ, 576, 745

Churazov, E., Forman, W., Jones, C., Bohringer, H. 2003, ApJ, 590, 225

Collinge, M. J., Brandt, W. N., Kaspi, S., et al. 2001, ApJ, 557, 2

Contini, M. 2004, MNRAS, 354, 675

Corral, A., Barcons, X., Carrera, F. J., Ceballos, M. T., \& Mateos, S. 2005, A\&A, 431,97

Czerny, B., Doroshenko, V. T., Nikołajuk, M., et al. 2003, MNRAS, 342, 1222 di Nella, H., Garcia, A. M., Garnier, R., \& Paturel, G. 1995, A\&AS, 113, 151

Elvis, M., Soltan, A., \& Keel, W. C. 1984, ApJ, 283, 479

Elvis, M., Wilkes, B. J. McDowell, J. C., et al. 1994, ApJS, 95, 1

Elvis, M., Risaliti, G., Nicastro, F., et al. 2004, ApJ, 615, L25

Eracleous, M., Shields, J. C., Chartas, G., \& Moran, E. C. 2002, ApJ, 565, 108

Fabian, A. C., Sanders, J. S., Crawford, C. S., et al. 2003, MNRAS, 344, L48

Falcke, H., Körding, E., \& Markoff, S. 2004, A\&A, 414, 895

Ferrarese, L. 2002, ApJ, 578, 90

Filho, M. E., Barthel, P. D., \& Ho, L. C. 2000, ApJS, 129, 93

Filho, M. E., Barthel, P. D., \& Ho, L. C. 2002, ApJS, 142, 223

Filippenko, A. V. \& Sargent, W. L. W. 1989, ApJ, 342, L11

Filippenko, A. V., Ho, L. C., \& Sargent, W. L. W. 1993, ApJ, 410, L75

Fiore, F., Giommi, P., Vignali, C., et al. 2001a, MNRAS, 327, 771

Fiore, F., Pellgrini, S., Matt, G., et al. 2001b, ApJ, 556, 150

Fruscione, A., Greenhill, L. J., Filippenko, A. V., et al. 2005, ApJ, 624, 103

Fukazawa, Y., Iyomoto, N., Kubota, A., Matsumoto, Y., \& Makishima, K. 2001, A\&A, 374, 73

Gebhardt, K., Richstone, D., Tremaine, S., et al. 2003, ApJ, 583, 92

Ghisellini, G., Haardt, F., \& Matt, G. 1994, MNRAS, 267, 743

Gonzalez Delgado, R. M., Perez, E., Tadhunter, C., Vilchez, J. M., \& RodriguezEspinosa, J. M. 1997, ApJS, 108, 155

Guainazzi, M., Matt, G., Brandt, W. N., et al. 2000, A\&A, 356, 463

Guainazzi, M., Marshall, W., \& Parmar, A. N. 2001, MNRAS, 323, 75

Guainazzi, M., Fabian, A. C., Iwasawa, K., Matt, G., \& Fiore, F. 2005, MNRAS, 356, 295

Haardt, F., \& Maraschi, L. 1991, ApJ, 380, L51

Heckman, T. M., Ptak, A., Hornschemeier, A., \& Kauffmann, G. 2005, ApJ, 634, 161

Ho, L. C. 1999 , ApJ, 516, 672

Ho, L. C. 2002, ApJ, 564, 120

Ho, L. C. 2003, Active Galactic Nuclei: From Central Engine to Host Galaxy, ASP Conf. Ser., 290, 379

Ho, L. C. \& Peng, C. Y. 2001, ApJ, 555, 650

Ho, L. C. \& Ulvestad, J. S. 2001, ApJS, 133, 77

Ho, L. C., Filippenko, A. V., \& Sargent, W. L. 1995, ApJS, 98, 477

Ho, L. C., Filippenko, A. V., \& Sargent, W. L. W. 1997a, ApJS, 112, 315

Ho, L. C., Filippenko, A. V., \& Sargent, W. L. W. 1997b, ApJ, 487, 568

Ho, L. C. Feigelson, E. D., Townsley, L. K., et al. 2001, ApJ, 549, L51

Irwin, J. A., Saikia, D. J., \& English, J. 2000, AJ, 119, 1592

Isobe, T., Feigelson, E. D., \& Nelson, P. I. 1986, ApJ, 306, 490

Iwasawa, K., Fabian, A. C., \& Matt, G. 1997, MNRAS, 289, 443

Iwasawa, K., Fabian, A. C., Almaini, O., et al. 2000, MNRAS, 318, 879

Iwasawa, K., Wilson, A. S., Fabian, A. C., \& Young, A. J. 2003, MNRAS, 345, 369

Iyomoto, N., Fukazawa, Y., Nakai, N., \& Ishihara, Y. 2001, ApJ, 561, L69

Kaastra, J. S., Steenbrugge, K. C., Raassen, A. J. J., et al. 2002, A\&A, 386, 427

Karachentsev, I. D. \& Sharina, M. E. 1997, A\&A, 324, 457

Kaspi, S., Smith, P. S., Netzer, H., Maoz, D., Jannuzi, B. T., \& Giveon, U. 2000, ApJ, 533, 631

Keel, W. C., \& Miller, J. S. 1983, ApJ, 266, L89
Kollmeier, J. A., et al. 2005, ApJ, sumbitted [arXiv: astro-ph/0508657]

Koratkar, A. P., \& Gaskell, C. M. 1991, ApJ, 370, L61

Kormendy, J. \& Richstone, D. 1995, ARA\&A, 33, 581

Kraemer, S. B., George, I. M., Crenshaw, D. M., \& Gabel, J. R. 2004, ApJ, 607, 794

Lamer, G., McHardy, I. M., Uttley, P., \& Jahoda, K. 2003, MNRAS, 338, 323

Lasota, J.-P., Abramowicz, M. A., Chen, X., et al. 1996, ApJ, 462, 142

Loewenstein, M., Mushotzky, R. F., Angelini, L., Arnaud, K. A., \& Quataert, E. 2001, ApJ, 555, L21

Magorrian, J., Tremaine, S., Richstone, D., et al. 1998, AJ, 115, 2285

Maiolino, R., Rieke, G.H., 1995, ApJ 454, 95

Maiolino, R., Salvati, M., Bassani, L., et al. 1998, A\&A, 338, 781

Maiolino, R., Comastri, A., Gilli, R., et al. 2003, MNRAS, 344, L59

Marconi, A., Risaliti, G., Gilli, R., et al. 2004, MNRAS, 351, 169

Martins, L. P., Viegas, S. M., \& Gruenwald, R. 2003, ApJ, 587, 562

Mas-Hesse, J. M., Rodriguez-Pascual, P. M., de Cordoba, L. S. F., \& Mirabel, I. F. 1994, ApJS, 92, 599

Mateos, S., Barcons, X., Carrera, F. J., et al. 2005, A\&A, 433, 855

Matt, G., Guainazzi, M., Frontera, F., et al., 1997, A\&A, 325, L13

Matt, G., Guainazzi, M., Maiolino, R., et al. 1999, A\&A, 341, L39

McElroy, D. B., 1995, ApJS, 100, 105

Merloni, A., Heinz, S., \& di Matteo, T. 2003, MNRAS, 345, 1057

Merritt, D., \& Ferrarese, L. 2001, MNRAS, 320, L30

Moran, E. C., Kay, L. E., Davis, M., Filippenko, A. V., \& Barth, A. J. 2001, ApJ, $556, \mathrm{~L} 75$

Mulchaey, J. S., Koratkar, A., Ward, M. J., et al. 1994, ApJ, 436, 586

Narayan, R., \& Yi, I. 1994, ApJ, 428, L13

Netzer, H., Chelouche, D., George, I. M., et al. 2002, ApJ, 571, 256

Nicastro, F., 2000, ApJ, 530, L65

Osterbrock, D. E. 1981, ApJ, 249, 462

Panessa, F., 2004, Ph.D. Thesis, University of Bologna, (http://venus.ifca.unican.es/ panessa/)

Panessa, F., \& Bassani, L. 2002, A\&A, 394, 435

Pappa, A., Geogantopoulos, I., Stewart, G. C., \& Zezas, A. L. 2001, MNRAS, 326, 995

Park, M.-G., \& Ostriker, J. P. 2001, ApJ, 549, 100

Paturel, G., Teerikorpi, P., Theureau, G., et al. 2002, A\&A, 389, 19

Pellegrini, S. 2005, ApJ, 624, 155

Pérez García, A. M., \& Rodríguez Espinosa, J. M. 2001, ApJ, 557, 39

Perez-Olea, D. E., \& Colina, L. 1996, ApJ, 468, 191

Pietsch, W. \& Read, A. M. 2002, A\&A, 384, 793

Pounds, K. A., Reeves, J. N., Page, K. L., et al. 2003, MNRAS, 341, 953

Ptak, A., Terashima, Y., Ho, L. C., \& Quataert, E. 2004, ApJ, 606, 173

Quataert, E., di Matteo, T., Narayan, R., \& Ho, L. C. 1999, ApJ, 525, L89

Richstone, D., Ajhar, E. A., Bender, R., et al. 1998, Nature, 395, A14

Risaliti, G., \& Elvis, M. 2004, Supermassive Black Holes in the Distant Universe, ASSL, 308, 187

Risaliti G., Maiolino R., \& Salvati M., 1999, ApJ, 522, 157

Sandage, A., \& Bedke, J. 1994, The Carnegie Atlas of galaxies. Volume I, Carnegie Institution of Washington

Sandage, A., Tammann, G. A., \& Yahil, A. 1979, ApJ, 232, 352

Schmitt, H. R., Donley, J. L., Antonucci, R. R. J., et al. 2003, ApJ, 597, 768

Shakura, N. I. \& Sunyaev, R. A. 1973, A\&A, 24, 337

Soldatenkov, D. A., Vikhlinin, A. A., \& Pavlinsky, M. N. 2003, Astron. Lett., 29, 298

Stetson, P. B., \& Gibson, B. K. 2001, MNRAS, 328, L1

Terashima, Y. \& Wilson, A. S. 2001, ApJ, 560, 139

Terashima, Y., \& Wilson, A. S. 2003, ApJ, 583, 145

Terashima, Y., Ho, L. C., Ptak, A. F. 2000, ApJ, 533, 729

Terashima, Y., Iyomoto, N., Ho, L. C., \& Ptak, A. F. 2002, ApJS, 139, 1

Tonry, J. L., Dressler, A., Blakeslee, J. P., et al. 2001, ApJ, 546, 681

Tremaine, S., Gebhardt, K., Bender, R., et al. 2002, ApJ, 574, 740

Tully, R. B. 1988 (Cambridge and New York: Cambridge University Press), 221

Turner, T. J., Mushotzky, R. F., Yaqoob, T., et al. 2002, ApJ, 574, L123

Ward, M. J., Done, C., Fabian, A. C., Tennant, A. F., \& Shafer, R. A. 1988, ApJ, 324, 767

Woo, J.-H., \& Urry, C. M. 2002, ApJ, 579, 530

Wrobel, J. M. 1991, AJ, 101, 127

Yaqoob, T., George, I. M., Nandra, K., et al. 2001, ApJ, 546, 759

Yuan, F., Markoff, S., Falcke, H., \& Biermann, P. L. 2002, A\&A, 391, 139 
F. Panessa et al.: On the X-ray, optical emission line and black hole mass properties of local Seyfert galaxies, Online Material $p 1$

\section{Online Material}




\section{Appendix: Notes on individual sources}

In this section we report notes on individual sources. We only discuss the X-ray data analyzed in this work; for those sources with X-ray data taken from the literature the references are reported in Table 2. We give a description of: (i) the nuclear X-ray morphologies (ii) the XMM-Newton and Chandra spectral results and (iii) results from the literature. Spectral best fit results are discussed only for spectra with more than 100 counts.

NGC 1058 - In both Chandra and XMM-Newton images a nuclear core is absent, in agreement with the absence of a radio core detection (Ho \& Ulvestad 2001). The upper limit on the 2-10 keV luminosity reported for this source has been derived from the Chandra observation assuming $\Gamma=1.8$, in agreement with the value reported by Ho et al. (2001) on the same data set. NGC 1275 - This object is the central galaxy of the Perseus cluster. The Chandra image shows a compact nucleus surrounded by a complex structure which has been extensively studied in recent years (Fabian et al. 2003, and reference therein). Since Chandra public data for the nucleus suffered from heavy pile-up, here we use for the spectral analysis an $X M M-N e w t o n$ observation taken from the archive. The $0.5-$ $10 \mathrm{keV}$ spectrum has been extracted from a region of $20^{\prime \prime}$ radius. The soft spectrum is clearly dominated by the diffuse thermal emission of the cluster; the $2-10 \mathrm{keV}$ spectrum is described by a power-law $(\Gamma=1.95 \pm 0.01)$ absorbed by the Galactic column density. A prominent $\mathrm{Fe} \mathrm{K} \alpha$ line associated with the cluster is present at $\sim 6.7 \mathrm{keV}$; another at $\sim 6.4 \mathrm{keV}$ having an equivalent width of $272 \pm 25 \mathrm{eV}$ is also visible and is likely associated with the active nucleus. The hard luminosity is in agreement with Churazov et al. (2003).

NGC 2683 - A low-luminosity unresolved radio core (Irwin et al. 2000) is coincident with the nuclear peak emission seen in the Chandra image. For this object, the $2-10 \mathrm{keV}$ flux has been derived from the Chandra data assuming $\Gamma=1.8$ and Galactic absorption.

NGC 3079 - See C06 for the analysis of the XMM-Newton data. The $10^{\prime \prime}$ region around the nucleus is resolved in the Chandra image: a strong nuclear source is embedded in a bubble of diffuse emission. A Chandra and HST study of the superbubble by Cecil et al. (2002) shows that the optical and $\mathrm{X}$-ray emissions match spatially. The radio core position is coincident with the $2-10 \mathrm{keV}$ peak. We extracted the spectrum from a circular region of $2^{\prime \prime}$ in radius. The spectral parameters are not well constrained due to the poor photon statistics, however the results are in good agreement with the XMM-Newton ones. We fit the data with an absorbed power-law $(\Gamma=1.71 \pm 0.5$, $N_{\mathrm{H}} \leq 4.6 \times 10^{21} \mathrm{~cm}^{-2}$ ). The strong FeK line at $6.4 \mathrm{keV}$ (detected at $>99 \%$ of significance with $X M M$-Newton ) suggests that this source is heavily absorbed and confirm the BeppoSAX results which indicate that the source is Compton thick (Iyomoto et al. 2001).

NGC 3147 - A Chandra snap-shot observation for this source has been published by Terashima \& Wilson (2003). The same data set is analyzed here. The $0.3-10 \mathrm{keV}$ image reveals a bright compact source surrounded by very faint soft and weak diffuse emission. The $2-10 \mathrm{keV}$ core is clearly detected and restricted to a region of $2^{\prime \prime}$ in radius. The nuclear spectrum was extracted from this region but it suffers from mild pile-up. The effect of pile-up has been corrected using the PILEUP model in XSPEC. The spectrum is described by a power-law $(\Gamma=1.88 \pm 0.15)$ modified by low absorption. These results are in agreement with those by Terashima \& Wilson (2003). The $2-10 \mathrm{keV}$ absorption corrected flux is a factor of 2.5 higher than what measured with ASCA.

NGC 3489 - The Chandra image for this source shows faint nuclear emission and an off-nuclear source within 5" from the nucleus. The upper limit on the $2-10 \mathrm{keV}$ flux has been derived assuming $\Gamma=1.8$ and Galactic absorption and it is in good agreement with the results reported in Ho et al. (2001). An upper limit on the radio detection is reported in Filho et al. (2002).

NGC 3516 - This galaxy has been extensively studied in $\mathrm{X}$-rays and it is known to be variable both in flux and spectrum (Guainazzi et al. 2001). We analyzed the zeroth-order image and spectrum of a $47 \mathrm{ks}$ ACIS/LETGS observation (Netzer et al. 2002) and a $100 \mathrm{ks}$ EPIC observation. In the Chandra image we find a bright point-like source at the nuclear position. Soft diffuse emission surrounds the nucleus and it extends for $10^{\prime \prime}$. The nuclear spectrum has been extracted from a circular region of $2^{\prime \prime}$ in radius, while we used 25" for the XMM-Newton spectrum. Both the XMM-Newton and Chandra spectra show a flat continuum with overimposed several narrow components of Fe $\mathrm{K} \alpha$ along with a broad line (see Turner et al. 2002). Here we adopt the Chandra results where $\Gamma=1.31 \pm 0.1$, $N_{\mathrm{H}} \sim 1 \times 10^{22} \mathrm{~cm}^{-2}$ and the equivalent width of the iron $\mathrm{K}$ line at $6.4 \mathrm{keV}$ is $96_{-0.57}^{+0.47} \mathrm{eV}$. The hard spectrum obtained is due to the fact that the source was in a low state at the time of the observation. Despite this, fluxes and luminosities are in agreement with previous measurements.

NGC 3608 - Only Chandra data are available for this object. The image of the nuclear region is characterized by a complex structure, where the peak of the emission is in the nucleus but is surrounded by off-nuclear sources. The $2-10 \mathrm{keV}$ flux has been obtained assuming a power-law with $\Gamma=1.8$ (fixed) and Galactic absorption. Only an upper limit on the radio detection is available (Wrobel 1991).

NGC 3627 - In the Chandra image some structures are visible close to the nucleus and in the XMM-Newton image the weak nuclear emission is comparable to a source off-set by $\sim 10^{\prime \prime}$. The upper limit on the Chandra 2-10 keV flux has been derived assuming a $\Gamma=1.8$ and is consistent with the result obtained by Ho et al. (2001) on the same data set. The XMM-Newton spectrum is probably contaminated.

NGC 3982 - This source has been observed by ASCA. We have derived the $2-10 \mathrm{keV}$ flux from the count rate presented in Moran et al. (2001) assuming a $\Gamma=1.8$ and Galactic absorption. An unresolved radio core has been detected (Ho \& Ulvestad 2001).

NGC 4051 - A previous Chandra HETG study for this source has been performed by Collinge et al. (2001). Here we use the ID 2148 observation (frame time $0.4 \mathrm{~s}$ ). The $2-10 \mathrm{keV}$ compact nucleus of this object is embedded in soft diffuse emission. An extraction radius of $2^{\prime \prime}$ allows us to reduce the contamination in the soft band. The spectrum suffers from mild pile-up $(\leq 11 \%)$, therefore we use the PILEUP model in XSPEC to take into account this effect (alpha parameter $=$ 0.29 ). We fitted the $0.5-10 \mathrm{keV}$ spectrum with a soft thermal component with $k T$ at $0.2 \mathrm{keV}$, a power-law plus an Fe line at $6.4 \mathrm{keV}$ significant at $>99 \%\left(E W=345_{-91}^{+49} \mathrm{eV}\right)$. The spectral index $\left(\Gamma=1.33_{-0.03}^{+0.7}\right)$ and the value of the column density $\left(\leq 1.4 \times 10^{20} \mathrm{~cm}^{-2}\right)$ are in good agreement with the spectral values obtained with $X M M-N e w t o n$ except for the Fe line which is weaker with $E W=240 \pm 40 \mathrm{eV}$ (C06). Our results are in agreement with what reported in Lamer et al. (2003), i.e. that the spectral parameters for both the continuum emission and the 
line are variable. A complex radio structure is present in this source (Ho \& Ulvestad 2001).

NGC 4235 - The ASCA hard X-ray luminosity has been obtained by modeling the spectrum with an absorbed power-law $\left(\Gamma=1.57 \pm 0.06, N_{\mathrm{H}}=1.5 \times 10^{21}\right)$. The ASCA data are taken from the HEASARC archive. An unresolved radio core is present in the source (Ho \& Ulvestad 2001).

NGC 4258 - The Chandra image of this object shows a prominent nuclear emission located at the same position of the nuclear radio core (Ho \& Ulvestad 2001). An extraction radius of $2^{\prime \prime}$ allows us to avoid the contamination of an off-nuclear source positioned at only $3^{\prime \prime}$ from the nucleus. This source is not resolved in the XMM-Newton image which is dominated by a hard point-like nucleus and unresolved diffuse emission (C06). The XMM-Newton and Chandra spectral results are in good agreement with results reported by Pietsch \& Read (2002). The Chandra spectral shape is a power-law with $\Gamma=1.4 \pm 0.1$ and an intrinsic absorption of $7 \times 10^{22} \mathrm{~cm}^{-2}$. No FeK $\alpha$ line is detected in this case. The XMM-Newton hard luminosity is a factor of 2 lower than the Chandra luminosity. However, it has been shown that both X-ray flux and spectral shape are highly variable in this source (Fruscione et al. 2005). The same is true for the iron $\mathrm{K} \alpha$ which had been detected in previous ASCA and BeppoSAX observations but which is not significantly detected both in our Chandra and XMM-Newton measurements.

NGC 4388 - Chandra observed this source on 2001 June (Iwasawa et al. 2003). The $2-10 \mathrm{keV}$ image shows a bright nucleus which appears embedded in diffuse emission in the full band image which extends for $\sim 20^{\prime \prime}$ and appears to be correlated with the optical ionization cones. We selected a circular region of $2^{\prime \prime}$ in radius centered on the hard peak position which agrees very well with the northern radio core supposed to be the true nucleus (Ho \& Ulvestad 2001). The spectrum is affected by mild pile-up. We fitted the hard strong component with an heavily absorbed power-law having a spectral index of $\sim 1.7$ and obtain a $N_{\mathrm{H}}=3.3 \times 10^{23} \mathrm{~cm}^{-2}$. A strong $\mathrm{Fe} \mathrm{K} \alpha$ line at $6.32 \mathrm{keV}$ is detected $(E W \sim 450 \mathrm{eV})$ while marginal is the detection of a line at $7.0 \mathrm{keV}$. The soft component is fitted with a power-law with $\Gamma=0.4 \pm 0.2$. Our spectral results are in good agreement with those reported in Iwasawa et al. (2003). The XMM-Newton spectrum is characterized by a flat spectral slope and the amount of absorption as well as the equivalent width of the iron line are in good agreement with the Chandra values. Variability in the column density has been reported by Elvis et al. (2004).

$N G C 4472$ - This is an elliptical giant galaxy. The XMMNewton image reveals strong soft diffuse emission. The Chandra higher angular resolution allows us to resolve the $25^{\prime \prime}$ region of XMM-Newton in diffuse emission and offnuclear sources around the optical nuclear position. The Chandra 2-10 keV image is characterized by a complex structure and there is no evidence for a dominant core emission (details on this Chandra data set are given in Loewenstein et al. 2001 and Soldatenkov et al. 2003). For this reason the hard X-ray flux and luminosity are treated as upper limits. This source is marginally detected in the radio band (Ho \& Ulvestad 2001).

NGC 4579 - This object has been observed for $\sim 35 \mathrm{ks}$ (Eracleous et al. 2002) and $\sim 3$ ks (Ho et al. 2001; Terashima \& Wilson 2003) by Chandra . Chandra images show a hard compact nucleus surrounded by soft diffuse emission which extends for $\sim 40^{\prime \prime}$. In both observations the nucleus is significantly piled-up $(\sim 11 \%)$. In Eracleous et al. (2002) the spectral fitting has been corrected from the pile-up effect by using the simulator-based forward-fitting tool LYNX, developed by the ACIS instrument team. The data are described by a simple power-law with no absorption in excess to the Galactic value $(\Gamma=1.88 \pm 0.03)$. Here we derive the $2-10 \mathrm{keV}$ fluxes and luminosities from the $3 \mathrm{ks}$ observation and find that they are in good agreement with Eracleous et al. (2002) and Ho et al. (2001) results. A radio core is detected (Ho \& Ulvestad 2001). NGC 5194 - This galaxy, also called M 51, has been observed by Chandra four times. A $15 \mathrm{ks}$ observation has been analyzed by Terashima \& Wilson (2001); here we consider the $26 \mathrm{ks}$ observation. The Chandra image shows a complex nuclear region characterized by extended features and off-nuclear sources. A bright nucleus is seen in the optical position coincident with the radio core position (Ho \& Ulvestad 2001) and it appears to be compact in the $2-10 \mathrm{keV}$ image. The nuclear spectrum has been extracted from a region of $2^{\prime \prime}$ in radius. The soft emission has been modeled with a thermal plasma with $k T=0.32 \pm 0.4 \mathrm{keV}$ while the hard component with a power-law having photon index fixed to $\Gamma=2$; the strong iron line detected at $\sim 6.4 \mathrm{keV}$ has an equivalent width greater than $2.6 \mathrm{keV}$, which is an indication of the Compton thick nature of this source. This has also been confirmed by a BeppoSAX observation of M 51 which has shown that the nucleus is absorbed by a column density of $5.6 \times 10^{24} \mathrm{~cm}^{-2}$ (Fukazawa et al. 2001).

NGC 5548 - This source has been observed by Chandra using the LETG (Kaastra et al. 2002) and the HETG (Yaqoob et al. 2001) instruments. The zeroth-order observations are affected by heavy pile-up. We analyzed the $92 \mathrm{ks} X M M$-Newton observation available from the archive. The Chandra and XMM-Newton images reveal the presence of a point-like bright nucleus. We extracted the XMM-Newton nuclear spectrum from a region of $25^{\prime \prime}$ in radius. A power-law with $\Gamma=1.69 \pm 0.01$ modified by Galactic absorption gives a good fit of the spectrum. An FeK line is detected $\left(E W=67_{-7}^{+37} \mathrm{eV}\right)$ plus a prominent soft excess. Our best fit is consistent with the work on the same data set by Pounds et al. (2003).

NGC 6482 - This source has been observed by Chandra for $\sim 20 \mathrm{ks}$. The nuclear emission is very bright, diffuse and typical of an elliptical galaxy. We extract the spectrum from a circular region of $2^{\prime \prime}$ in radius centered in the optical position which is coincident with the $2-10 \mathrm{keV}$ peak. The spectrum is dominated by a soft thermal component $(k T=0.8 \pm 0.5 \mathrm{keV})$, while the hard component has been modelled with an absorbed $\left(\leq 1.45 \times 10^{21} \mathrm{~cm}^{-2}\right)$ power-law having the photon index fixed to 1.8. This source has not been detected at $8.4 \mathrm{GHz}$ (Filho et al. 2002).

NGC 6503 - This galaxy has been observed by Chandra twice (exposures of $2 \mathrm{ks}$ and $13 \mathrm{ks}$ ). Here we analyze the longer exposure observation. The nuclear position is determined by the comparison of the Chandra image with the HST image. The nucleus has been detected, although it is very weak. Four off-nuclear sources have also been detected. Few counts are extracted from a region of $2^{\prime \prime}$ in radius from which flux and luminosity have been derived assuming $\Gamma$ fixed to 1.8. Emission has not been detected in the radio band (Filho et al. 2000).

$N G C 7479$ - We report the analysis of the $50 \mathrm{ks} X M M-$ Newton observation of this source (Iwasawa et al. in preparation). From a comparison between the EPIC images and the DSS image we clearly detect the nuclear source which appears to be fainter than the few off-nuclear sources associated with the asymmetrical spiral arms. The nuclear spectrum has been extracted from a circular region of $20^{\prime \prime}$ in order to avoid contamination. However, the $2-10 \mathrm{keV}$ image reveals the possible presence of unresolved sources within $20^{\prime \prime}$ around the 
F. Panessa et al.: On the X-ray, optical emission line and black hole mass properties of local Seyfert galaxies, Online Material p 4 nucleus. To model the continuum we fixed the photon index of the power-law to 1.8 and left the column density free to vary $\left(N_{\mathrm{H}}=5.8 \times 10^{23} \mathrm{~cm}^{-2}\right)$. We also fit the soft component using a thermal model $(k T=2.0 \pm 0.5 \mathrm{keV})$. The radio core is unresolved (Ho \& Ulvestad 2001). 